\title{
Bayesian Identification of Soil Stratigraphy based on Soil Behaviour Type Index
}

\begin{tabular}{|r|l|}
\hline Journal: & Canadian Geotechnical Journal \\
\hline Manuscript ID & cgj-2017-0714.R1 \\
\hline Manuscript Type: & Article \\
\hline Complete List of Authors: & $\begin{array}{l}\text { Cao, Zi-Jun; Wuhan University, State Key Laboratory of Water Resources } \\
\text { and Hydropower Engineering Science } \\
\text { Zheng, Shuo; Wuhan University, State Key Laboratory of Water Resources } \\
\text { and Hydropower Engineering Science } \\
\text { Li, Dianqing; Wuhan University, State Key Laboratory of Water Resources } \\
\text { and Hydropower Engineering Science } \\
\text { Phoon, Kok-Kwang; National University of Singapore, Department of Civil \& } \\
\text { Environmental Engineering }\end{array}$ \\
\hline Keyword: & $\begin{array}{l}\text { Cone Penetration Test, Soil Stratification, Bayesian Approach, Soil } \\
\text { Behaviour Type Index, Subset Simulation }\end{array}$ \\
\hline $\begin{array}{r}\text { Is the invited manuscript for } \\
\text { consideration in a Special } \\
\text { Issue? : }\end{array}$ & \begin{tabular}{l} 
Not applicable (regular submission) \\
\hline
\end{tabular} \\
\hline
\end{tabular}




\title{
Submitted to Canadian Geotechnical Journal
}

\section{Bayesian Identification of Soil Stratigraphy based on Soil Behaviour Type Index}

\author{
Zi-Jun Cao ${ }^{1}$, Shuo Zheng ${ }^{2}$, Dian-Qing Li ${ }^{3}$, Kok-Kwang Phoon ${ }^{4}$
}

\begin{abstract}
${ }^{1}$ Associate Professor, State Key Laboratory of Water Resources and Hydropower Engineering Science, Institute of Engineering Risk and Disaster Prevention, Wuhan University; 8 Donghu South Road, Wuhan 430072, P. R. China; (t) 86-27-6877 4036 (f) (86)-27-6877 4295 (e) zijuncao@,whu.edu.cn (corresponding author)
\end{abstract}

${ }^{2} \mathrm{Ph} . \mathrm{D}$. Candidate, State Key Laboratory of Water Resources and Hydropower Engineering Science, Institute of Engineering Risk and Disaster Prevention, Wuhan University; 8 Donghu South Road, Wuhan 430072, P. R. China; (t) 86-27-6877 4036 (f) (86)-27-6877 4295 (e) zhengshuo@whu.edu.cn

${ }^{3}$ Professor, State Key Laboratory of Water Resources and Hydropower Engineering Science, Institute of Engineering Risk and Disaster Prevention, Wuhan University; 8 Donghu South Road, Wuhan 430072, P. R. China. (t) 86-6877 2496 (f) (86)-27-6877 4295 (e) dianqing@whu.edu.cn

${ }^{4}$ Distinguished Professor, Department of Civil and Environmental Engineering, National University of Singapore, Blk E1A, \#07-03, 1 Engineering Drive 2, Singapore 117576, Singapore. (t) 65-6516 6783 (f) 65-6779 1635 (e) kkphoon@nus.edu.sg 


\begin{abstract}
Cone penetration test (CPT) has been widely used to determine the soil stratigraphy (including the number $N$ and thicknesses $\underline{H}_{N}$ of soil layers) during geotechnical site investigation because it is rapid, repeatable, and economical. For this purpose, several deterministic and probabilistic approaches have been developed in the literature, but these approaches generally only give the "best" estimates (e.g., the most probable values) of $N$ and $\underline{H}_{N}$ based on CPT data according to prescribed soil stratification criteria, providing no information on the identification uncertainty (degrees-of-belief) in these "best" estimates. This paper develops a Bayesian framework for probabilistic soil stratification based on the profile of soil behaviour type index $I_{c}$ calculated from CPT data. The proposed Bayesian framework not only provides the most probable values of $N$ and $\underline{H}_{N}$, but also quantifies their associated identification uncertainty based on the $I_{c}$ profile and prior knowledge. Equations are derived for the proposed approach, and they are illustrated and validated using real and simulated $I_{c}$ profiles. Results show that the proposed approach properly identifies the most probable soil stratigraphy based on the $I_{c}$ profile and prior knowledge, and rationally quantifies the uncertainty in identified soil stratigraphy with consideration of inherent spatial variability of $I_{c}$.
\end{abstract}

Key Words: Cone Penetration Test; Soil Stratification; Bayesian Approach; Soil Behaviour Type Index; Subset Simulation 


\section{Introduction}

Cone penetration tests (CPTs) provide a versatile method for geotechnical site investigation, during which a cylindrical steel probe is pushed into the ground to measure mechanical responses of soils during penetration, such as cone tip resistance $q_{c}$, sleeve friction $f_{s}$, and pore water pressure $u$, etc. (e.g., Lunne et al. 1997; Mayne et al. 2002). In the past few decades, CPT has been gaining increasing use worldwide because it is rapid, repeatable, and economical, and provides nearly continuous measurements over the depth (e.g., Robertson 2009 , 2016). One of primary uses of CPT is to identify the soil stratigraphy based on its measurements, such as $q_{c}$ and $f_{s}$. In general, this consists of two major steps: (1) determine the soil type at each testing depth (i.e., soil classification) based on CPT measurements, yielding a profile of the soil type with the depth; and (2) identify the number $N$ and thicknesses (or boundaries) $\underline{H}_{N}=\left[H_{1}, H_{2}, \ldots, H_{N}\right]$ of soil layers based on the soil type profile generated in the first step (i.e., soil stratification).

Because no soil samples are recovered from CPTs for visual inspection and laboratory testing, CPT-based soil classification is frequently accomplished through some soil classification system established from past observations and engineering experience, such as Robertson soil behaviour type (SBT) charts (e.g., Robertson and Campanella 1983a,b; Robertson 1990, 2009, 2016) and Unified Soil Classification System (USCS) (e.g., Zhang and Tumay 1999; Kurup and Griffin 2006; ASTM 2011). This can be performed either in a deterministic way (e.g., Douglas and Olsen 1981; Robertson and Campanella 1983a,b; Robertson 1990; Jefferies and Davies 1993; Olsen and Mitchell 1995; Eslami and Fellenius 1997; Robertson and Wride 1998; Robertson 2009,2016; Ganju et al. 2017) or under a probabilistic framework (e.g., Zhang and Tumay 1999; Tumay et al. 2008; Jung et al. 2008; Cetin and Ozan 2009; Wang et al. 2013; Depina et al. 2015). Among various CPT-based soil classification systems, the SBT index $I_{c}$ is widely used, which is essentially the radius of 
concentric circles approximating boundaries of SBTs in soil classification charts and locating CPT data points on the chart to determine their corresponding SBTs (Jefferies and Davies 1993; Robertson and Wride 1998; Robertson 2009). $I_{c}$ is defined as a function of CPT measurements (e.g., $q_{c}, f_{s}$, and $u$ ), and it provides a concrete and effective concept for classifying soils according to their mechanical behaviours and allows automated identification of the SBT for each CPT data points (Ku et al. 2010; Ching et al. 2015b; Li et al. 2015). However, due to spatial variability of soils, CPT measurements at different depths vary spatially, so are $I_{c}$ values calculated from CPT data, even for soils with the same SBT (Uzielli et al. 2005). The spatial variability of $I_{c}$ poses a profound challenge in identifying soil stratigraphy (i.e., determining $N$ and $\underline{H}_{N}$ ) from a single profile of $I_{c}$ with certainty. Engineering judgment is, hence, often exercised, in a subjective and unquantifiable manner, to stratify soil layers based on the $I_{c}$ profile, which might give inconsistent soil stratigraphy by different engineers due to their different experience, expertise, and judgments. More importantly, the uncertainty (or degrees-of-belief) in soil stratigraphy inferred from the $I_{c}$ profile in such a subjective and unquantifiable way is unknown.

Several approaches have been developed for soil stratification using CPT data in an objective and quantitative way, such as $\mathrm{T}$ ratio method (e.g., Wickremesinghe and Campanella 1991), clustering method (e.g., Hegazy and Mayne 2002; Liao and Mayne 2007), statistical analysis using modified Bartlett statistics (e.g., Phoon et al. 2003, 2004), wavelet transform modulus maxima (WTMM) method (Ching et al. 2015b), and Bayesian methods (e.g., Cao and Wang 2013; Wang et al. 2013,2017). These approaches are able to provide the "best" estimates of $N$ and $\underline{H}_{N}$ in terms of some prescribed criterion for soil stratification, but they provide little information on the uncertainty in estimated $N$ and $\underline{H}_{N}$. Such an uncertainty might have significant impacts on geotechnical analyses and designs (e.g., Wang et al. 2010a; Phoon et al. 2013; Ching et al. 2013, 2015a) and, hence, needs to be properly quantified. 
This paper develops a Bayesian framework for probabilistic soil stratification based on the profile of $I_{c}$. Under the proposed Bayesian framework, the inherent spatial variability of $I_{c}$ along the depth is taken into account through random field modeling, and the uncertainty in $N$ and $\underline{H}_{N}$ estimated from the $I_{c}$ profile is explicitly quantified using their posterior distributions, reflecting the degrees-of-belief in their estimates. Bayesian framework is attracting increasing interest in geotechnical engineering. For example, it has been successfully applied to selection of empirical models between water retention curve parameters and particle-size distribution (Chiu et al. 2012), soil stratification model class selection (e.g., Cao and Wang 2013; Wang et al. 2013; Houlsby and Houlsby 2013) and identification of random field models of soil properties (e.g., Wang et al. 2010b; Ching et al. 2015c; Huang et al. 2017b).

Solving posterior distributions is an essential step in Bayesian analysis, which is a non-trivial task when the posterior distribution involves high dimensional integrals (e.g., Beck and Au 2002; Cao and Wang 2013; Wang et al. 2013; Ching et al. 2015b). To address this issue, an advanced Monte Carlo simulation (MCS) technique called Subset Simulation (SuS) (Au and Beck 2001, 2003; DiazDelaO et al. 2017) is applied in the proposed approach to evaluate Bayesian equations based on a recently proposed concept called Bayesian Updating with Structural Reliability Method (BUS) (Straub and Papaioannou 2015). The paper starts with random field modeling of the $I_{c}$ profile, followed by development of the Bayesian framework and its key components. Then, the computational difficulty in solving Bayesian equations is addressed using BUS with $\mathrm{SuS}$, and the implementation procedure of the proposed approach is described. Finally, the proposed approach is illustrated and validated using real-life and simulated $I_{c}$ profiles. 


\section{Random Field Modeling of Soil Behaviour Type Index Profile}

Consider, for example, a sounding of CPT results measured at an interval of $\Delta \mathrm{D}$, based on which the $I_{c}$ value at each measuring depth is calculated in accordance with its definitions proposed in the literature (e.g., Jefferies and Davies 1993; Robertson and Wride 1998; Robertson 2009). Without loss of generality, the definition of $I_{c}$ developed by Robertson and Wride (1998) and Robertson (2009) is adopted in this paper for development of the proposed approach, which is written as:

$$
I_{c}=\sqrt{\left(3.47-\log _{10} Q_{t n}\right)^{2}+\left(\log _{10} F_{r}+1.22\right)^{2}}
$$

where $Q_{t n}=\left[\left(q_{t}-\sigma_{v o}\right) / P_{a}\right]\left(P_{a} / \sigma_{v o}^{\prime}\right)^{n}$ is the normalized tip resistance; $q_{t}=$ cone tip resistance corrected for unequal end-area effects; $\sigma_{v o}, \sigma_{v o}^{\prime}$, and $P_{a}$ are the vertical total stress, vertical effective stress, and atmospheric pressure (i.e., $100 \mathrm{kPa}$ ), respectively; $n=0.381\left(I_{c}\right)+0.05\left(\sigma_{v o}^{\prime} / P_{a}\right)-0.15$ is the stress exponent accounting for SBT and $\sigma_{v o}^{\prime}$ (Robertson 2009); $F_{r}=\left[f_{s} /\left(q_{t}-\sigma_{v o}\right)\right] 100 \%$ is the normalized friction ratio. Using Eq. (1), the $I_{c}$ value at each testing depth is calculated from its corresponding CPT measurements (e.g., $q_{t}$ and $f_{s}$ ), yielding a profile of $I_{c}$ over the depth.

Based on the $I_{c}$ value calculated from Eq. (1), soils are classified into six SBTs (see Table 1), which approximately range from Zone 2 to Zone 7 in Robertson SBT chart shown in Figure 1. The bold solid lines shown in Figure 1 indicate the boundaries between different SBTs based on $I_{c}$. Note that the $I_{c}$ value from Eq. (1) increases as $Q_{t n}$ decreases and $F_{r}$ increases. Hence, the minimum, $I_{c}$,min, and maximum, $I_{c, \max }$, of $I_{c}$ occur at the upper-left $\left(F_{r}=\right.$ $\left.0.1, Q_{t n}=1000\right)$ and bottom-right $\left(F_{r}=10, Q_{t n}=1\right)$ corners of Robertson SBT chart shown in Figure 1 , respectively, and their values are $I_{c, \min }=0.52$ and $I_{c, \max }=4.12$. The $I_{c}$ values typically vary over the depth within the range from $I_{c}$,min to $I_{c, \text { max }}$. For example, Figures 2(a) and 2(b) show, respectively, a set of $q_{t}$ and $f_{s}$ data over the depth obtained from National Geotechnical Experimentation Site (NGES) at Texas A\&M University (Zhang and Tumay, 
1999; Briaud, 2000; Wang et al. 2013). Based on these $q_{t}$ and $f_{s}$ data, a profile of $I_{c}$ at the NGES is calculated using Eq. (1), as shown in Figure 2(c), where the boundaries between different SBTs based on the $I_{c}$ are shown by vertical dashed lines. The $I_{c}$ value shown in Figure 2(c) varies from 1.8 to 3.2, and its corresponding SBT ranges from SBT 3 (Clays: silty clay to clay) to SBT 6 (Sands: clean sand to silty sand). The spatial variability of $I_{c}$ with the depth can be observed even for soils with the same SBT, such as those belonging to SBT 5 below the depth of $7.0 \mathrm{~m}$. Such spatial variability unavoidably affects the soil stratification results (e.g., $N$ and $\underline{H}_{N}$ ) inferred from the $I_{c}$ profile and the degrees-of-belief (or uncertainty) in these estimates.

To explicitly incorporate the spatial variability of $I_{c}$ into CPT based-soil stratification, random field theory (Vanmarcke, 2010) is used in this study to model the $I_{c}$ profile. Then, as shown in Figure 3, a profile of $I_{c}$ over the depth of $H$ is considered to be obtained from $N$ statistically homogenous soil layers and is represented by $N$ one-dimensional and mutually independent lognormal random fields $\underline{I}_{\underline{c}}, n=1,2, \ldots, N$. The random field $\underline{I}_{\underline{c} n}$ for the $n$-th soil layer consists of a number, $k_{n}$, of spatially correlated lognormal random variables with a mean $\mu_{n}$ and standard deviation $\sigma_{n}$, which represent the $I_{c}$ values at $k_{n}$ testing depths in the $n$ th soil layer. In the context of random field theory, the spatial correlation between variations of $I_{c}$ (or, equivalently, the logarithm $\ln I_{c}$ of $I_{c}$ that follows Gaussian distribution in this study) at different depths is specified by a correlation function. Here, the correlation function of $\ln I_{c}$ is taken as a single exponential correlation function that is frequently used in geotechnical analysis (e.g., Fenton, 1999a, b; Phoon et al. 2003, 2004; Uzielli et al. 2005; Li et al. 2014; Qi and Li, 2018; Xiao et al. 2018). The correlation coefficient $\rho_{n}$ between the logarithms (i.e., $\ln I_{c}\left(Z_{i}\right)$ and $\left.\ln I_{c}\left(Z_{j}\right)\right)$ of $I_{c}$ at respective depths $Z_{i}$ and $Z_{j}$ is then given by:

$\rho_{n}\left[\ln I_{c}\left(Z_{i}\right), \ln I_{c}\left(Z_{j}\right)\right]=\exp \left(-2\left|d_{i, j}\right| / \lambda_{n}\right)$ 
where $d_{i, j}=\left|Z_{i}-Z_{j}\right|\left(i, j=1,2, \ldots, k_{n}\right)$ is a distance between depths $Z_{i}$ and $Z_{j}$ within the $n$-th layer; $\lambda_{n}=$ scale of fluctuation of $\ln I_{c}$ in the $n$-th soil layer, and it is a separation distance within which the correlation among $\ln I_{c}$ values at different depths is relatively strong.

To completely define the $N$ random fields of $I_{c}$ shown in Figure 3, information on $N$, $\underline{H}_{N}$, and $\underline{\theta}_{n}=\left[\mu_{n}, \sigma_{n}, \lambda_{n}\right](n=1,2, \ldots, N)$ are needed. Herein, it shall be pointed out that, for the purpose of soil stratification that focuses on identifying the number and boundaries of soil layers, only $N$ and $\underline{H}_{N}$ are of intrinsic interest in this study. Nevertheless, $\underline{\theta}_{n}$ are needed to explicitly model inherent spatial variability of $I_{c}$ for soil stratification. They are treated as nuisance parameters in the study, which are quantities necessarily included in the formulation and analysis but of no intrinsic interest (Sivia and Skilling 2006; Wang et al. 2013; Cao and Wang 2014), as discussed in the next section.

\section{Bayesian Framework for Identification of Soil Stratigraphy}

For a given profile of $I_{c}$, there are many possible combinations of $N$ and $\underline{H}_{N}$, each of which corresponds to a soil stratification model. The occurrence probability of each soil stratification model (i.e., a combination of $N$ and $\underline{H}_{N}$ ) given the $I_{c}$ (or, equivalently, $\ln I_{c}$ ) data is quantified by their joint probability density function (PDF) $p\left(\underline{H}_{N}, N \mid \underline{\xi}\right)$, where $\underline{\xi}=\left[\xi_{1}\right.$, $\left.\xi_{2}, \ldots, \xi_{N}\right]$ is a set of $\ln I_{c}$ data obtained from the $N$ soil layers and $\xi_{n}=\left[\ln I_{c}\left(Z_{1}\right), \ln I_{c}\left(Z_{2}\right), \ldots\right.$, $\left.\ln I_{c}\left(Z_{k n}\right)\right](n=1,2, \ldots, N)$ represent a number $k_{n}$ of $\ln I_{c}$ values obtained at $k_{n}$ depths in the $n$-th soil layer. Then, the most probable soil stratigraphy, which is specified by the most probable number $N^{*}$ of soil layers and their most probable thicknesses $\underline{H}_{N^{*}}^{*}=\left[H_{1}^{*}, H_{2}^{*}, \ldots, H_{N^{*}}^{*}\right]$ given a $I_{c}$ profile, can be probabilistically reasoned according to $p\left(\underline{H}_{N}, N \mid \xi\right)$. More importantly, the $p\left(\underline{H}_{N}, N \mid \xi\right)$ provides information on the uncertainty in $N$ and $\underline{H}_{N}$ given $\underline{\xi}$. Such information, quantitatively and explicitly, reflects how reliable the soil stratigraphy identified from $\underline{\xi}$ is. 
Note that the number of dimensions of $p\left(\underline{H}_{N}, N \mid \underline{\xi}\right)$ is equal to $N+1$, and it increases as $N$ increases. Direct solving such a PDF with varying dimensions is a non-trivial task. Alternatively, based on the conditional probability (Ang and Tang 2007), $p\left(\underline{H}_{N}, N \mid \xi\right)$ can be re-written as:

$p\left(\underline{H}_{N}, N \mid \underline{\xi}\right)=p\left(\underline{H}_{N} \mid \underline{\xi}, N\right) P(N \mid \underline{\xi})$

where $p\left(\underline{H}_{N} \mid \xi, N\right)$ is the conditional PDF of $\underline{H}_{N}$ based on $\underline{\xi}$ and a given soil stratification model with $N$ soil layers, and it has $N$ dimensions that is considered as a fixed condition; $P(N \mid \xi)$ is conditional probability of $N$ given $\xi$, and it has only one dimension. As indicated by Eq. (3), identification of soil stratigraphy can be divided into two steps: (1) compare the soil stratification models with different numbers (e.g., $N$ ) of soil layers based on $P(N \mid \xi)$ and determine $N^{*}$ among a number of possible $N$ values; and (2) evaluate $p\left(\underline{H}_{N} \mid \underline{\xi}, N\right)$ for quantifying the uncertainty in $\underline{H}_{N}$ based on $\underline{\xi}$ for a given soil stratification model with $N$ (e.g., $\left.N=N^{*}\right)$ soil layers and determine their most probable thicknesses $\underline{H}_{N}^{*}=\left[H_{1}^{*}, H_{2}^{*}, \ldots, H_{N}^{*}\right]$ and boundaries. These two steps are introduced in the following two subsections, respectively.

\section{Comparing soil stratification models with different numbers of soil layers}

Let $N_{\max }$ denote the maximum number of soil layers contained in a profile of $I_{c}$. Then, $N$ is defined as a discrete random variable ranging from 1 to $N_{\max }$. Using Bayes' Theorem, $P(N \mid \xi)$ is written as (Yan et al. 2009; Yuen, 2010a,b; Cao and Wang 2013; Wang et al. 2013, 2014):

$$
P(N \mid \underline{\xi})=p(\underline{\xi} \mid N) P(N) / p(\underline{\xi})
$$

where $P(N)=$ prior probability of $N$ reflecting the prior knowledge on $N$ in the absence of CPT data; $p(\underline{\xi})=\sum_{N=1}^{N_{\max }} p(\underline{\xi} \mid N) P(N)$ is a normalizing constant and is independent of $N ; p(\underline{\xi} \mid N)$ $=$ conditional PDF of $\xi$ given the soil stratification model with $N$ layers, and it is frequently referred to as the "evidence" for the soil stratification model with $N$ layers provided by $\xi$. In 
the case of no prevailing prior knowledge on $N$, the $N_{\max }$ possible values (i.e., $1,2, \ldots, N_{\max }$ ) of $N$ are considered having the same prior probability, i.e., $P(N)=1 / N_{\max }$. Then, based on Eq. (4), $P(N \mid \xi)$ is proportional to the evidence $p(\xi \mid N)$, which means that maximizing $p(\xi \mid N)$ with respect to $N$ leads to the maximum value of $P(N \mid \xi)$ and, hence, $N^{*}$. Calculation of $p(\xi \mid N)$ is pivotal to evaluating Eq. (4) for quantifying uncertainty in $N$ based on $\xi$ and determining $N^{*}$.

Using the Theorem of Total Probability (Ang and Tang, 2007), $p(\xi \mid N)$ is calculated as: $p(\underline{\xi} \mid N)=\int p\left(\underline{\xi}^{\mid} \underline{H}_{N}, N\right) p\left(\underline{H}_{N} \mid N\right) d \underline{H}_{N}$

where $p\left(\underline{H}_{N} \mid N\right)=$ prior distribution of $\underline{H}_{N}$ given the soil stratification model with $N$ layers, and it quantifies the prior knowledge on $\underline{H}_{N}$ in the absence of $\xi$; and $p\left(\xi \mid \underline{H}_{N}, N\right)=$ conditional joint PDF of $\xi$ given a soil stratification model consisting of $N$ layers with thicknesses of $\underline{H}_{N}$. Under a Bayesian framework, $p\left(\underline{\xi} \mid \underline{H}_{N}, N\right)$ is referred to as the "likelihood function" that is a function of $\underline{H}_{N}$ given $\underline{\xi}$ and $N$, and it quantitatively reflects the model fit with $\underline{\xi}$. Before moving to formulations of $p\left(\underline{H}_{N} \mid N\right)$ and $p\left(\underline{\xi} \underline{H}_{N}, N\right)$ required for evaluating the evidence in Eq. (5), the next subsection discusses how to quantify uncertainty in $\underline{H}_{N}$ of the soil stratification model with $N$ layers based on $\xi$ and prior knowledge under a Bayesian framework.

\section{Quantifying uncertainty in soil stratigraphy for a given number of soil layers}

In this subsection, $\xi$ is considered to be acquired from $N$ soil layers, where $N$ is a fixed value and is used as a condition for inferring $\underline{H}_{N}$ from $\underline{\xi}$ according to $p\left(\underline{H}_{N} \mid \underline{\xi}, N\right)$. Within a Bayesian framework, $p\left(\underline{H}_{N} \mid \xi, N\right)$ is referred to as the posterior distribution of $\underline{H}_{N}$ based on $\xi$, and it is expressed as :

$p\left(\underline{H}_{N} \mid \underline{\xi}, N\right)=p\left(\underline{\xi} \mid \underline{H}_{N}, N\right) p\left(\underline{H}_{N} \mid N\right) / p(\underline{\xi} \mid N)$

The $p\left(\underline{H}_{N} \mid \xi, N\right)$ in Eq. (6) quantifies the uncertainty in layer thicknesses $\underline{H}_{N}$ (or, equivalently, layer boundaries) of the soil stratification model with $N$ layers based on CPT data (i.e., $\xi$ ) and 
prior knowledge. It involves the likelihood function $p\left(\xi \mid \underline{H}_{N}, N\right)$, prior distribution $p\left(\underline{H}_{N} \mid N\right)$, and a normalizing constant $p(\xi \mid N)$ independent of $\underline{H}_{N}$ for a given $N$ value, which is the evidence for the soil stratification model with $N$ layers (see Eq. (5)). Formulations of $p\left(\underline{H}_{N} \mid N\right)$ and $p\left(\xi \mid \underline{H}_{N}, N\right)$ are provided in the next two subsections, respectively.

\section{Prior distribution of soil layer thicknesses}

In the case of no prevailing prior knowledge on soil layer thicknesses $\underline{H}_{N}=\left[H_{1}, H_{2}, \ldots, H_{N}\right]$, they can be considered uniformly distributed, each of which ranges from 0 to CPT sounding depth $H$, i.e., $0<H_{n}<H$ for $n=1,2, \ldots, N$. Nevertheless, it is not straightforward to formulate a joint uniform prior distribution of $\underline{H}_{N}$ because they have to satisfy a linear constraint, i.e., $\sum_{n=1}^{N} H_{n}=H$, which enforces the correlation among thicknesses of different soil layers. Alternatively, this study formulates $p\left(\underline{H}_{N} \mid N\right)$ from a geometric perspective. Consider, for example, there are two soil layers (i.e., setting $N=2$ ) within a testing depth of $15 \mathrm{~m}$. Their possible thicknesses are constrained by the linear equation $H_{1}+H_{2}=15$, as shown in Figure 4 . In other words, all the combinations of $H_{1}$ and $H_{2}$ lie on the off-diagonal solid line shown in Figure 4. If there is no preference on any combinations of $H_{1}$ and $H_{2}$ based on prior knowledge, the combinations of $H_{1}$ and $H_{2}$ are uniformly distributed on the off-diagonal solid line. Such reasoning can be generalized to $N$ dimensional space for $\underline{H}_{N}$. Similarly, all the possible combinations of $\underline{H}_{N}$ are uniformly distributed within a $N-1$ dimensional simplex $\Omega=$ $\left\{\sum_{n=1}^{N} H_{n}=H, 0<H_{n}<H\right\}$ with the length of all edges equal to $\sqrt{2} H$, which is a subspace of the hyperplane defined by $\sum_{n=1}^{N} H_{n}=H$ and is constrained by $\left\{0<H_{n}<H, n=1,2, \ldots, N\right\}$. Such an uniform distribution of $\underline{H}_{N}$ can be derived from a flat Dirichlet distribution that represents a uniform prior distribution of normalized soil layer thicknesses $H_{n} / H, n=1,2, \ldots, N$, belonging to a $N-1$ dimensional standard simplex $\Omega_{\mathrm{S}}=\left\{\sum_{n=1}^{N} H_{n} / H=1,0<H_{n} / H<1\right\}$ formed from unit vectors and with the edge length of $\sqrt{2}$. Within $\Omega_{\mathrm{S}}$, the joint uniform PDF of $H_{n} / H$, 
$n=1,2, \ldots, N$, is calculated from the Gamma function evaluated at $N$, i.e., $\Gamma(N)$, by the definition of the flat Dirichlet distribution (Bishop 2006); otherwise, their joint PDF is equal to zero. From a geometric perspective, $\Omega$ can be obtained by extending each edge of $\Omega_{\mathrm{S}}$ by $H$ times from $\sqrt{2}$ to $\sqrt{2} H$, by which the probability space is enlarged by $H^{N-1}$ times because there are $N-1$ free uncertain parameters among the $N$ soil layer thicknesses under the linear constrain $\sum_{n=1}^{N} H_{n}=H$. To ensure the normalization of the uniform prior distribution within $\Omega$, the joint PDF of $\underline{H}_{N}$ is obtained by reducing the joint PDF of normalized soil layer thicknesses by $H^{N-1}$ times, and is expressed as:

$$
p\left(\underline{H}_{N} \mid N\right)=\left\{\begin{array}{cc}
\Gamma(N) / H^{N-1} & \text { for } \underline{H}_{N} \in\left[\sum_{n=1}^{N} H_{n}=H, 0<H_{n}<H\right] \\
0 & \text { otherwise }
\end{array}\right.
$$

where $H^{N-1}$ serves as a normalizing constant. As indicated by Eq. (7), $p\left(\underline{H}_{N} \mid N\right)$ is a constant for a given $N$ value and testing depth $H$.

\section{Likelihood function}

The likelihood function $p\left(\xi \mid \underline{H}_{N}, N\right)$ quantifies information on $\underline{H}_{N}$ of the soil stratification model with $N$ soil layers provided by $\xi$. As discussed in section entitled "Random Field Modeling of Soil Behaviour Type Index Profile", this study models the $I_{c}$ profile by $N$ mutually independent lognormal random fields $\underline{I}_{\underline{c} n}(Z), n=1,2, \ldots, N$ (see Figure 3 ). Correspondingly, the profile of $\ln I_{c}$ (i.e., $\xi=\left[\xi_{1}, \xi_{2}, \ldots, \xi_{N}\right]$ ) obtained from the $N$ soil layers are considered as a realization of the $N$ random fields with model parameters $\underline{\theta}_{n}=\left[\mu_{n}, \sigma_{n}, \lambda_{n}\right], n$ $=1,2, \ldots, N$. Then, $p\left(\underline{\xi} \mid \underline{H}_{N}, N\right)$ is expressed as (Cao and Wang 2013; Houlsby and Houlsby 2013):

$$
p\left(\underline{\xi} \mid \underline{H}_{N}, N\right)=\prod_{n=1}^{N} p\left(\underline{\xi}_{n} \mid \underline{H}_{N}, N\right)
$$


where $p\left(\xi_{n} \mid \underline{H}_{N}, N\right), n=1,2, \ldots, N$ is the likelihood function for the $n$-th soil layer. As indicated by Eq. (8), the likelihood function for the soil stratification model with $N$ soil layers is a function of $\underline{H}_{N}$ for a given set of $\underline{\xi}$ because the division of $\underline{\xi}$ into $\underline{\xi}_{1}, \underline{\xi}_{2}, \ldots$, and $\underline{\xi}_{N}$ relies on $\underline{H}_{N}$. Nevertheless, it shall be pointed out that CPT data are measured at an interval of $\Delta \mathrm{D}$. If the change of $\underline{H}_{N}$ is very small in comparison with $\Delta \mathrm{D}$, the division of $\underline{\xi}$ into $\underline{\xi}_{1}, \underline{\xi}_{2}, \ldots$, and $\underline{\xi}_{N}$ may not be affected by the change of $\underline{H}_{N}$, leading to a constant likelihood function with $\underline{H}_{N}$. As the change of $\underline{H}_{N}$ increases and becomes sufficiently large to affect the division of $\xi$, there will be a sudden jump in the value of likelihood function, indicating that the likelihood function is a discontinuous function of $\underline{H}_{N}$.

Using the Theorem of Total Probability, $p\left(\underline{\xi}_{n} \mid \underline{H}_{N}, N\right)$ is written as:

$$
p\left(\underline{\xi}_{n} \mid \underline{H}_{N}, N\right)=\int p\left(\underline{\xi}_{n} \mid \underline{\theta}_{n}, \underline{H}_{N}, N\right) p\left(\underline{\theta}_{n} \mid \underline{H}_{N}, N\right) d \underline{\theta}_{n}
$$

where $p\left(\underline{\xi}_{n} \mid \underline{\theta}_{n}, \underline{H}_{N}, N\right)$ is a joint Gaussian PDF of $\underline{\xi}_{n}$ for a given set of random field parameters $\underline{\theta}_{n}=\left[\mu_{n}, \sigma_{n}, \lambda_{n}\right]$ in the $n$-th soil layer, and it needs the information on $\underline{H}_{N}$ and $N$ to divide $\underline{\xi}$ into $\underline{\xi}_{n}, n=1,2, \ldots, N$; and $p\left(\underline{\theta}_{n} \mid \underline{H}_{N}, N\right)$ is the prior distribution of $\underline{\theta}_{n}$ in the $n$-th soil layer for a given soil stratification model comprised of $N$ soil layers with layer thicknesses equal to $\underline{H}_{N}$.

For a given set of $\underline{\theta}_{n}$, the joint Gaussian PDF $p\left(\underline{\xi}_{n} \mid \underline{\theta}_{n}, \underline{H}_{N}, N\right)$ of $\underline{\xi}_{n}$ is given by:

$$
p\left(\underline{\xi}_{n} \mid \underline{\theta}_{n}, \underline{H}_{N}, N\right)=\left(2 \pi \sigma_{\mathrm{ln}, n}^{2}\right)^{-\frac{k_{n}}{2}}\left|\operatorname{det} \underline{R}_{n}\right|^{-\frac{1}{2}} \exp \left\{-\frac{1}{2 \sigma_{\mathrm{ln}, n}^{2}}\left[\underline{\xi}_{n}-\mu_{\mathrm{ln}, n} \underline{l}_{n}\right]^{T} \underline{R}_{n}^{-1}\left[\underline{\xi}_{n}-\mu_{\mathrm{ln}, n} \underline{l}_{n}\right]\right\}
$$

where $\mu_{\ln , n}=\ln \mu_{n}-\sigma_{\ln , n}{ }^{2} / 2$ and $\sigma_{\ln , n}=\sqrt{\ln \left[1+\left(\sigma_{n} / \mu_{n}\right)^{2}\right]}$ are the mean and standard deviation of $\ln I_{c}$ in the $n$-th soil layer; $\underline{R}_{n}$ is the correlation matrix of $\xi_{n}$, and its $(i, j)$-th entry represents the correlation coefficient $\rho_{n}\left[\ln I_{c}\left(Z_{i}\right), \ln I_{c}\left(Z_{j}\right)\right]$ of $\ln I_{c}$ values at respective depths $Z_{i}$ and $Z_{j}$, which is given by Eq. (2); and $\underline{l}_{n}$ is a column vector with $k_{n}$ components that are all equal to one. 
Although only $\underline{H}_{N}$ and $N$ are of intrinsic interest in this study, the information on $\underline{\theta}_{n}, n$ $=1,2, \ldots, N$, is needed to formulate the likelihood function (see Eqs. (8)-(10)), and they are treated as nuisance parameters and are dealt with through marginalization in Eq. (9). Without prevailing information on $\underline{\theta}_{n}$ available at a site, the marginalization can be performed over typical ranges of $\underline{\theta}_{n}$ (Sivia and Skilling 2006). Then, $p\left(\underline{\theta}_{n} \mid \underline{H}_{N}, N\right)$ is simply taken as a joint uniform prior distribution of $\underline{\theta}_{n}$ defined by their typical ranges, which is written as:

$$
p\left(\underline{\theta}_{n} \mid \underline{H}_{N}, N\right)=\left\{\begin{array}{cc}
\frac{1}{\left(\mu_{n, \text { max }}-\mu_{n, \text { min }}\right)\left(\sigma_{n, \text { max }}-\sigma_{n, \text { min }}\right)\left(\lambda_{n, \text { max }}-\lambda_{n, \text { min }}\right)} & \text { for } \mu_{n} \in\left[\mu_{n, \text { min }}, \mu_{n, \text { max }}\right], \\
\sigma_{n} & \in\left[\sigma_{n, \text { min }}, \sigma_{n, \text { max }}\right], \\
\lambda_{n} & \in\left[\lambda_{n, \text { min }}, \lambda_{n, \text { max }}\right] \\
0 & \text { otherwise }
\end{array}\right.
$$

where $\mu_{n, \min }, \sigma_{n, \min }$, and $\lambda_{n, \min }$ are the respective lower bounds of $\mu_{n}, \sigma_{n}$, and $\lambda_{n}$; and $\mu_{n, \max }$, $\sigma_{n, \max }$, and $\lambda_{n, \max }$ are their respective upper bounds.

Substituting Eqs. (7)-(11) into Eqs. (5) and (6) gives the $p(\xi \mid N)$ for comparing soil stratification models with different numbers $N$ of soil layers and the $p\left(\underline{H}_{N} \mid \underline{\xi}, N\right)$ for quantifying uncertainty in $\underline{H}_{N}$ of a given soil stratification model with a fixed number (i.e., $N$ ) of soil layers based on $\xi$ and prior knowledge. Note that the $p(\xi \mid N)$ is also involved in $p\left(\underline{H}_{N} \mid \xi, N\right)$ (see Eq. (6)). Solving the $p(\xi \mid N)$ and $p\left(\underline{H}_{N} \mid \xi, N\right)$ is a key step to determine the soil stratigraphy and its associated identification uncertainty. This is a non-trivial task in this study because of the discontinuity of the likelihood function with respect to $\underline{H}_{N}$, constraint relationship among soil layer thicknesses (i.e., $\sum_{n=1}^{N} H_{n}=H$ ), and high-dimensional integral involved in the evidence and the posterior distribution (see Eqs. (5) and (6), respectively), particularly as $N$ is relatively large (say $N>5$ ). These computational difficulties defeat most of inference techniques for Bayesian updating, such as conjugate distributions, Laplace asymptotic approximate, and Metropolis-Hastings algorithm, etc. Recently, some advanced methods, such as transitional Markov Chain Monte Carlo (TMCMC) algorithm (Ching and Chen 2007; Yuen 2010b; Ching and Wang 2016) and BUS with SuS (Straub and 
Papaioannou 2015; DiazDelaO et al. 2017), are developed for handling computational difficulties involved in Bayesian analysis. Both TMCMC and BUS with SuS are feasible in solving high-dimensional Bayesian updating problems and, more importantly, they are able to estimate the evidence for Bayesian model class selection. However, to the best of our knowledge, capacity and performance of TMCMC for Bayesian updating problems involving a constrained probability space remain unexplored in the literature. In contrast, $\mathrm{Li}$ and $\mathrm{Au}$ (2010) and $\mathrm{Li}$ and Cao (2016) demonstrated that SuS provides a rational tool to explore a constrained probability space. This study implements BUS with SuS to, simultaneously, calculate $p(\xi \mid N)$ and $p\left(\underline{H}_{N} \mid \xi, N\right)$ in the next section.

\section{Bayesian Updating with Subset Simulation}

BUS (Straub and Papaioannou 2015; Straub et al. 2016) stems from the idea that converts Bayesian updating problems into equivalent reliability analysis problems by constructing an auxiliary observation domain $F$ using the likelihood function. By this means, reliability analysis methods (such as first order reliability method, direct MCS, importance sampling, line sampling, and SuS, etc.) can be used in Bayesian analyses to evaluate the posterior distribution (e.g., Eq. (6)) and model evidence (e.g., Eq. (5)) for model updating and comparison, respectively. In the context of BUS, the $F$ in this study is defined as (Straub and Papaioannou 2015; DiazDelaO et al. 2017):

$$
F=\left[c p\left(\underline{\xi} \mid \underline{H}_{N}, N\right)-U>0\right]
$$

where $c=$ a positive scalar constant ensuring $c p\left(\underline{\xi} \mid \underline{H}_{N}, N\right) \leq 1 ; p\left(\underline{\xi} \mid \underline{H}_{N}, N\right)=$ likelihood function given by Eq. (8); and $U=$ a uniform random variable ranging from 0 to unity and it is independent of $\underline{H}_{N}$. Using Eq. (12), $p\left(\underline{\xi} \mid \underline{H}_{N}, N\right)$ is re-written as (Straub 2011; Straub and Papaioannou 2015) 
$p\left(\underline{\xi} \mid \underline{H}_{N}, N\right)=c^{-1} \int_{0}^{1} I\left[\left(\underline{H}_{N}, U\right) \in F\right] d U$

where $I\left[\left(\underline{H}_{N}, U\right) \in F\right]=$ an indicator function. If $\left(\underline{H}_{N}, U\right)$ belongs to $F, I\left[\left(\underline{H}_{N}, U\right) \in F\right]$ is equal to one; otherwise, it is taken as 0. Substituting Eq. (13) into Eq. (5) gives

$p(\underline{\xi} \mid N)=c^{-1} \int_{\underline{H}_{N}} \int_{0}^{1} I\left(\left[\underline{H}_{N}, U\right] \in F\right) p\left(\underline{H}_{N} \mid N\right) d U d \underline{H}_{N}$

Since $U$ and $\underline{H}_{N}$ are independent and the PDF $p(U)$ of $U$ is equal to unity, the integral over the space of $U$ and $\underline{H}_{N}$ in Eq. (14) gives the occurrence probability (i.e., $\left.P(F)\right)$ of $F$, i.e.,

$p(\underline{\xi} \mid N)=c^{-1} P(F)$

Hence, evaluation of $p(\underline{\xi} \mid N)$ in Bayesian analysis can be achieved by calculating $P(F)$ in the equivalent reliability analysis problem, where uncertainty parameters $\underline{H}_{N}$ follow the prior distribution.

Moreover, using Eq. (6), the $F$ defined by Eq. (12) is re-written as:

$F=\left\{U<\frac{p\left(\underline{H}_{N} \mid \underline{\xi}, N\right)}{[c p(\underline{\xi} \mid N)]^{-1} p\left(\underline{H}_{N} \mid N\right)}\right\}$

where $[c p(\underline{\xi} \mid N)]^{-1}$ is a constant independent of $\underline{H}_{N}$. The terms $p\left(\underline{H}_{N} \mid \underline{\xi}, N\right)$ and $p\left(\underline{H}_{N} \mid N\right)$ in Eq. (16) can be viewed as target and sampling distributions in the context of rejection principle (Au and Wang 2014; Straub and Papaioannou 2015). Using the rejection principle, it is reasoned that the samples of $\underline{H}_{N}$ generated from $p\left(\underline{H}_{N} \mid N\right)$ and conditional on $F$ follow $p\left(\underline{H}_{N} \mid \underline{\xi}, N\right)$. In other words, $p\left(\underline{H}_{N} \mid \underline{\xi}, N\right)$ can be numerically represented by generating samples conditional on $F$ from the prior distribution $p\left(\underline{H}_{N} \mid N\right)$ under the BUS framework.

Nevertheless, as indicated in Eqs. (15) and (16), using the above formulation of BUS to evaluate the $p(\underline{\xi} \mid N)$ and to generate posterior samples of $\underline{H}_{N}$ from the prior distribution needs to specify $c$, the admissible values of which satisfy $c p\left(\xi \mid \underline{H}_{N}, N\right) \leq 1$. Determination of $c$ 
for BUS might not be a trivial task because of the complexity (e.g., high-dimensionality) in the likelihood function (e.g., Betz et al. 2014; DiazDelaO et al. 2017). To address this issue, DiazDelaO et al. (2017) proposed an alternative BUS algorithm, by which BUS is implemented with SuS without the need of predetermining $c$ by redefining $F$ in Eq. (12) as:

$$
F=\left\{\ln \left[\frac{p\left(\underline{\xi} \mid \underline{H}_{N}, N\right)}{U}\right]>-\ln c\right\}
$$

Then, the term $Y=\ln \left[p\left(\underline{\xi} \mid \underline{H}_{N}, N\right) / U\right]$ is used as the target response in SuS to generate conditional samples of $\underline{H}_{N}$ within $F$ (i.e., posterior samples of $\underline{H}_{N}$ under the BUS framework) from the prior distribution and to calculate the $P(F)$. SuS is an advanced MCS technique that evaluates the occurrence probability of a rare event of interest as a product a sequence of intermediate events with larger conditional probabilities $p_{0}(\mathrm{Au}$ and Beck 2001,2003; Li and Au 2010; Au and Wang 2014). It provides a robust yet efficient method to explore the rare event (e.g., $F$ ) and to calculate its occurrence probability (e.g., $P(F)$ ) by simulating intermediate events level by level, for which a number, $N_{c s}$, of conditional samples are generated in each level to evaluate their corresponding conditional probabilities. In addition, using the alterative BUS algorithm with $\mathrm{SuS}$, the value of $c$ is determined in an adaptive manner as SuS proceeds (DiazDelaO et al. 2017), which allows evaluating the evidence using Eq. (15). In this study, the alternative BUS algorithm proposed by DiazDelaO et al. (2017) is applied to generating posterior samples of $\underline{H}_{N}$ to numerically represent $p\left(\underline{H}_{N} \mid \underline{\xi}, N\right)$ for quantifying uncertainty in $\underline{H}_{N}$ and to calculating the evidence $p(\underline{\xi} \mid N)$ (i.e., $\left.c^{-1} P(F)\right)$ for comparing soil stratification models with different numbers of soil layers.

The alternative BUS algorithm with $\mathrm{SuS}$ progressively approaches to $F$ and determines posterior samples in $F$ based on the characteristic trend of the variation of $P(F)$ as a function of $-\ln c$. As the $P(F)$ changes from a slowly decreasing function of $-\ln c$ to a 
straight line with a slope of -1 , the minimum value of $-\ln c$ of posterior samples is determined, which corresponds to the largest admissible value $c_{\max }$ of $c$, i.e., the reciprocal of the maximum likelihood function, for BUS. Hence, in theory, the alternative BUS algorithm with SuS proposed by DiazDelaO et al. (2017) is able to generate posterior samples with the maximum likelihood function, which are identical to the most probable posterior values (MPPV) in the case of using a constant prior distribution (e.g., Eq. (7)). Nevertheless, in implementation, the $P(F)$ is estimated on a sample basis, and its estimate may randomly deviate around the theoretical characteristic trend, leading to random fluctuation in estimated $c_{\max }$ and MPPV. Such a random fluctuation can be reduced by increasing the number (i.e., $N_{c s}$ ) of samples generated in each SuS level. With a large number (i.e., $\left.N_{c s}=100,000\right)$ of samples, it is assumed that $\mathrm{SuS}$ provides reasonably accurate estimates of $P(F)$ and $c_{\text {max }}$, and generates random samples clustering around the MPPV in this study. For the sake of conciseness, detailed algorithm and implementing procedures of the alterative BUS formulation with SuS are not provided herein. Interested readers are referred to DiazDelaO et al. (2017) for details.

\section{Implementation Procedures}

Figure 5 shows the implementation procedure of the proposed approach schematically, which involve nine steps. Calculation details of each step and their associated equations are summarized as follow:

1. Obtain a set of CPT data and calculate the $I_{c}$ value at each testing depth using Eq. (1);

2. Choose a maximum number $N_{\max }$ of soil layers for the set of CPT data, leading to $N_{\max }$ possible values of $N$ varying from 1 to $N_{\max }$;

3. Determine a set of prior knowledge on random field parameters used to model the spatial variability of $I_{c}$, such as their typical ranges (i.e., $\left[\mu_{n, \min }, \mu_{n, \max }\right],\left[\sigma_{n, \min }, \sigma_{n, \max }\right]$, and $\left[\lambda_{n, \min }\right.$, $\left.\left.\lambda_{n, \max }\right]\right)$ needed in Eq. (11); 
4. Set $N=1$, for which the soil layer thickness is a deterministic value of $H$. Hence, both the prior and posterior distributions (i.e., $p\left(\underline{H}_{N} \mid N\right)$ and $\left.p\left(\underline{H}_{N} \mid \underline{\xi}, N\right)\right)$ for $N=1$ are equal to unity. Then, the evidence $p(\xi \mid N)$ for $N=1$ is calculated using Eqs. (5) and (8)-(11). Note that the 3-dimentional integration in Eq. (9) is evaluated numerically over the space of random field parameters in this study;

5. Construct the likelihood function $p\left(\xi \mid \underline{H}_{N}, N\right)$ using Eqs. (8)-(11) and the auxiliary observation domain $F$ defined by Eq. (17) for BUS for $N=2,3, \ldots, N_{\max }$, respectively;

6. Determine the conditional probability $p_{0}$ (typically chosen as 0.1 ) and the number, $N_{c s}$, of samples per subset stage for performing SuS to generate posterior samples of $\underline{H}_{N}$ and to calculate the $p(\xi \mid N)$ (i.e., $c^{-1} P(F)$ ) for the soil stratification model with $N$ layers, and then calculate $P(N \mid \xi)$ using Eq. (4). Note that the samples of $\underline{H}_{N}$ generated from prior distribution given by Eq. (7) shall be uniformly distributed within the simplex $\Omega=$ $\left\{\sum_{n=1}^{N} H_{n}=H, 0<H_{n}<H\right\}$. Since the linear constraint $\sum_{n=1}^{N} H_{n}=H$ is equivalent to the constraint $\sum_{n=1}^{N-1} H_{n}<H$ for $0<H_{n}<H, n=1,2, \ldots, N-1$, this is achieved by generating independent uniform random samples of $H_{n}, n=1,2, \ldots, N-1$, ranging from 0 to $H$ and using the $\sum_{n=1}^{N-1} H_{n}$ as the target response in $\mathrm{SuS}$ to drive the sampling space to $\Omega$ that is equivalently defined as $\left\{\sum_{n=1}^{N-1} H_{n}<H, 0<H_{n}<H\right\}$. For each sample of $H_{n}, n=1,2, \ldots, N$ 1 , its corresponding $H_{N}$ is calculated as $H-\sum_{n=1}^{N-1} H_{n}$. By this means, a large number of prior samples of $\underline{H}_{N}$ is first generated using SuS. Starting from the prior samples, SuS proceeds to explore $F$ for generating posterior samples of $\underline{H}_{N}$ and to determine $P(F)$ and $c$ for evaluating $p(\xi \mid N)$ (i.e., $\left.c^{-1} P(F)\right)$, during which all the samples remain within $\Omega$. Moreover, for each sample of $\underline{H}_{N}$ generated by $\operatorname{SuS}$ for exploring $F$, it needs to calculate the likelihood function $\left.p(\xi) \quad \underline{H}_{N}, N\right)$ for evaluating the target response $Y=\ln \left[p\left(\underline{\xi} \mid \underline{H}_{N}, N\right) / U\right]$. As indicated in Eqs. (8) and (9), there are $N$ 3-dimentional 
integrations involved in the likelihood function, which are, again, calculated numerically in this study.

7. Repeat steps 5 and 6 for $N=2,3, \ldots, N_{\max }$ to obtain their corresponding $p(\xi \mid N)$ and $P(N \mid \xi)$

8. Compare the values of $P(N \mid \xi)$ (or, equivalently, $p(\xi \mid N)$ in the case of using uniform prior probability of $N$ ) for different $N$ values and select the one with the maximum value of $P(N \mid \xi)$ as $N^{*}$. Note that the $N_{\max }$ values of $P(N \mid \xi)$ give the probability mass function (PMF) of $N$ based on the $I_{c}$, which quantify identification uncertainty in $N$;

9. Calculate the statistics (e.g., mean values $\underline{\mu}_{H}$, standard deviations $\underline{\sigma}_{H}$, and the MPPV $\underline{H}_{N^{*}}^{*}$ ) of $\underline{H}_{N}$ based on their posterior samples generated by $\operatorname{SuS}$ for $N=N^{*}$, among which $\underline{H}_{N^{*}}^{*}$ reflects the most probable soil stratigraphy identified from the proposed approach based on the $I_{c}$ profile and $\underline{\sigma}_{H}$ reflects its identification uncertainty. In this study, the $\underline{H}_{N^{*}}^{*}$ are taken as the posterior sample of $\underline{H}_{N^{*}}$ with the maximum value of the posterior distribution $p\left(\underline{H}_{N^{*}} \mid \xi, N^{*}\right)$ for $N=N^{*}$ or, equivalently, the maximum value of the likelihood function $p\left(\xi \mid \underline{H}_{N^{*}}, N^{*}\right)$ for $N=N^{*}$ because the prior distribution of soil layer thicknesses is a constant (see Eq.(7)). Note that the values of $p\left(\underline{\xi} \mid \underline{H}_{N^{*}}, N^{*}\right)$ corresponding to posterior samples have been calculated for evaluating the target response $Y$ in Step 6 as $N=N^{*}$, during which repetitive evaluations of the integral in Eq. (9) are needed. After the posterior samples are obtained, the MPPV $\underline{H}_{N^{*}}^{*}$ are identified with relative ease by comparing the values of $p\left(\underline{\xi} \mid \underline{H}_{N^{*}}, N^{*}\right)$ of posterior samples because a uniform prior distribution is adopted in this study and it is assumed the number of posterior samples are sufficiently large to generate samples clustering around MPPV.

Note that $\mathrm{SuS}$ is used under the BUS framework to generate posterior samples of $\underline{H}_{N}$ and to calculate $p(\xi \mid N)$ for each possible $N$ value in proposed approach. SuS already has 
several successful applications in geotechnical engineering (e.g., Au et al. 2010; Wang et al. 2010a, 2011; Santoso et al. 2011; Ahmed and Soubra 2014; Li et al. 2016a,b; Xiao et al. 2016; Huang et al. 2017a). It has been implemented in EXCEL (e.g., Au et al. 2010; Au and Wang 2014) and MATLAB (Li and Cao 2016) by developing toolboxes or user-functions. For the sake of briefness, detailed algorithms and implementation procedures of SuS is not introduced in this paper. Details of the SuS algorithm, its implementation procedure, and MATLAB codes for exploring an event (e.g., F) within a constrained probability space (e.g., $\Omega$ in this study) are refer to $\mathrm{Li}$ and $\mathrm{Au}(2010)$ and $\mathrm{Li}$ and Cao (2016).

In addition, to facilitate the practical implementation of the proposed approach, it can also be programmed as a user-function or toolbox in computer software, such as MATLAB (Mathworks Inc. 2017). Then, the users only need to provide prior knowledge and CPT test data (or $I_{c}$ profile) as input, and the user-function or toolbox will return the estimated soil stratigraphy (i.e., $N^{*}$ and $\underline{H}_{N^{*}}^{*}$ ) and its associated identification uncertainty (i.e., degrees-ofbelief) as output. By this means, the user-function or toolbox is treated as a "black box", with which geotechnical practitioners can use the proposed approach with relative ease. The proposed approach and its implementation procedures are illustrated and validated using reallife and simulated CPT data in the next two sections, respectively.

\section{Illustrative Example}

For illustration, the proposed approach is applied to identifying the soil stratigraphy at the clay site of the NGES at Texas A\&M University based on a set of CPT data obtained from the site, which is shown in Figure 2. The set of CPT data have been used to illustrate CPTbased soil classification and/or stratification approaches in the literature, including fuzzybased soil classification (FBSC) approach (Zhang and Tumay 1999), Bayesian soil classification and stratification (BSCS) approach based on Robertson SBT chart (Wang et al. 
2013), and the wavelet transform modulus maxima (WTMM) method for soil stratification (Ching et al. 2015b). Based on the boring log obtained from the ground surface to a depth of around $15.0 \mathrm{~m}$ (see Figure 6), the site consists of a sequence of sandy clay, clay, silty clay, and clay with silt seams (Zhang and Tumay 1999), and the groundwater table is at about 6.0 $\mathrm{m}$ below the ground surface. However, it shall be emphasized that the actual soil stratigraphy is unknown at a real site. Although in-situ boring and sampling allow inferring soil stratigraphy from soil samples, such an inference relies on the criterion adopted to delineate soil layer boundaries, which is somehow subjective because engineering experience and judgments are often exercised in a vague and unquantifiable manner.

In this study, the $I_{c}$ profile calculated from the set of CPT data (see Figure 2) is used as input in the proposed approach to identify the soil stratigraphy at the clay site. Consider, for example, that there are, to the maximum, 10 soil layers within the testing depth, i.e., $N_{\max }$ $=10$. The possible number (i.e., $N$ ) of soil layers therefore varies from 1 to 10 . Since the number and thicknesses of soil layers are unknown, there is not sufficient information on $\underline{\theta}_{n}$, $n=1,2, \ldots, N$, to justify some informative and sophisticated prior distribution for each soil layer. In this study, the prior knowledge of $\underline{\theta}_{n}$ is represented by a joint uniform prior distribution given by Eq. (11), where typical ranges of $\mu_{n}, \sigma_{n}$, and $\lambda_{n}$ are needed, and it is assumed identical for all soil layers. Without prevailing information on $\underline{\theta}_{n}$, it is suggested choosing ranges of $\underline{\theta}_{n}$ as wide as possible to define the uniform prior distribution so that it properly applies to all the layers. As discussed in section entitled "Random Field Modeling of Soil Behaviour Type Index Profile", the $I_{c}$ value typically ranges from $I_{c, \min }=0.52$ to $I_{c, \max }=$ 4.12 (see Figure 1). Such a range is simply taken as the typical range of $\mu_{n}$ in this study. For $\sigma_{n}$, its theoretical lower bound shall be 0 , indicating that no uncertainty exists in $I_{c}$. To the other extreme, the upper bound of $\sigma_{n}$ are considered to occur as the $I_{c}$ is uniformly distributed within $[0.52,4.12]$, and then $\sigma_{n, \max }$ is taken as the standard deviation corresponding to the 
uniform distribution of $I_{c}$, i.e., $\sigma_{n, \max }=(4.12-0.52) / 2 \sqrt{3}=1.04$ (Wang et al. 2013, 2014). In addition, the possible values of $\lambda_{n}$ are considered to range from $0.1 \mathrm{~m}$ (i.e., $\lambda_{n, \min }=0.1 \mathrm{~m}$ ) to $1.2 \mathrm{~m}$ (i.e., $\lambda_{n, \max }=1.2 \mathrm{~m}$ ), which are consistent with those reported in the literature (e.g., Uzielli et al. 2005). Table 2 summarizes the ranges of $\underline{\theta}_{n}$ adopted in this study, which are considered sufficiently wide to cover the typical values of random field parameters of $I_{c}$. It is recommended to adopt these ranges of $\underline{\theta}_{n}$ to define the prior distribution when using the proposed approach unless more informative prior distributions with narrower ranges can be well justified based on prior knowledge (Cao et al. 2016).

Using the prior knowledge and the $I_{c}$ profile shown in Figure 2 (c), the proposed approach gives the value of $p(\xi \mid N)$ (or, equivalent, $P(N \mid \xi)$ in the case of using uniform prior probability of $N$ ) for each possible $N$ value varying from 1 to 10 and generates the posterior samples of $\underline{H}_{N}$ to numerically represent $p\left(\underline{H}_{N} \mid \xi, N\right)$ using SuS, in which $p_{0}=0.1$ and $N_{c s}=$ 100,000. Based on the $p(\xi \mid N)$ values and posterior samples of $\underline{H}_{N}, N^{*}$ and $\underline{H}_{N^{*}}^{*}$ are determined for soil stratification. For each set of posterior samples of $\underline{H}_{N}$, the corresponding depths $\underline{D}_{N}=\left[D_{1}, D_{2}, \ldots, D_{N}\right]$ of soil layer lower boundaries can be calculated, and their most probable values $\underline{D}_{N^{*}}^{*}=\left[D_{1}^{*}, D_{2}^{*}, \ldots, D_{N^{*}}^{*}\right]$ are determined from $\underline{H}_{N^{*}}^{*}$ accordingly. More importantly, the uncertainty (or degrees-of-belief) in the identified soil stratigraphy is quantitatively reflected by the posterior probability $P(N \mid \xi)$ of $N$ and the posterior statistics (e.g., standard deviation) of $\underline{H}_{N}$ (or $\underline{D}_{N}$ ) evaluated from their posterior samples. Note that the soil stratification along the depth can be uniquely described by either soil layer thicknesses or layer boundary depths, which are equivalent to each other. This study formulates the soil layer thicknesses as uncertain parameters in lieu of layer boundary depths because the relative locations of different layer boundaries need to be considered when using them as updating parameters, leading to more complicated constrains (i.e., $D_{1}<D_{2}<\ldots<D_{N-2}<D_{N-}$ ${ }_{1}<D_{N}=H$ ) on layer boundary depths than those on soil layer thicknesses. 


\section{Most probable number of soil layers based the $I_{c}$ profile at the clay site}

Table 3 summarizes the logarithms (i.e., $\ln p(\xi \mid N)$ and $\ln P(N \mid \xi)$ ) of the evidence and posterior probability for each possible value of $N$ obtained from the proposed approach at the NGES site. The value of $\ln p(\xi \mid N)$ increases 304.99 to 352.93 as $N$ increases from 1 to 6 , and then it decreases from 352.93 to 337.11 as $N$ further increases from 6 to 10 , which is shown in Figure 7 as well. Similar trend is observed from the value of $\ln P(N \mid \underline{\xi})$ since the uniform prior probability of $N\left(\right.$ i.e., $\left.P(N)=1 / N_{\max }\right)$ is used in Eq. (4) in this example. It is found that the soil stratification model with six layers has the maximum values of $\ln p(\xi \mid N)$ and $\ln P(N \mid \xi)$. Hence, the most probable number of soil layers at the clay site is six, i.e., $N^{*}=6$, and its corresponding occurrence probability $P(N \mid \underline{\xi})$ for $N=6$ given the set of $I_{c}$ profile is about 0.533. It is also noted that the value of $\ln P(N \mid \xi)$ for $N=7$ is equal to -0.86 that corresponds to $P(N=7 \mid \xi)=0.423$, which are close to those (i.e., -0.63 and 0.533 ) for $N^{*}=6$. Then, it can be reasoned that the occurrence probabilities of other $N$ values (i.e., $N=1,2,3,4,5,8,9$, and 10) are less than 0.05 and are, hence, marginal. In other words, based on the set of $I_{c}$ profile shown in Figure 2(c) and prior knowledge, it is almost certain that there are 6 or 7 soil layers at the clay site. Nevertheless, the occurrence probability (i.e., 0.533 ) of $N^{*}=6$ is not overwhelmingly large (e.g., greater than 0.9 ) in comparison with that (i.e., 0.423) of $N=7$, leading to somewhat ambiguity in $N$ in this example. Such an ambiguity emphasizes the necessity of quantifying identification uncertainty (i.e., degrees-in-belief) in $N$.

\section{Most probable thicknesses of soil layers and their associated identification uncertainty}

Figure 8 shows the posterior samples of thicknesses (i.e., $H_{n}, n=1,2, \ldots, 6$ ) of the six soil layers generated by BUS with $\mathrm{SuS}$ based on the $I_{c}$ profile and prior knowledge in the top 
right-hand panels. Using these posterior samples, the marginal posterior PDFs of $H_{n}, n=1$, $2, \ldots, 6$, and the correlation coefficient $\rho_{m, n}$, where $m, n=1,2, \ldots, 6$ and $m \neq n$, between thicknesses of different soil layers are estimated, which are shown in the diagonal and bottom left-hand panels in Figure 8, respectively. They reflect the 6-dimensional joint posterior distribution $p\left(\underline{H}_{N^{*}} \mid \xi, N^{*}\right)$ of $H_{n}, n=1,2, \ldots, 6$ in a tractable and visual manner, which quantify the identification uncertainty associated with thicknesses of the six soil layers based on the $I_{c}$ profile shown in Figure 2(c) and prior knowledge. Figure 8 also shows the respective MPPVs (i.e., $\left.H_{n}^{*}, n=1,2, \ldots, 6\right)$ of soil layer thicknesses for $N^{*}=6$ determined from their joint posterior distributions by open red circles in the diagonal panels. It is found that the MPPVs of $H_{n}, n=1,2, \ldots, 6$ determined from the joint posterior distribution are not identical to those based on their respective marginal posterior distributions where the peak values occur. This is attributed to the fact that the soil layer thicknesses for $N^{*}=6$ are not globally identifiable in this example, which is confirmed by bimodal distributions of posterior samples (see top righthand panels).

Based on the joint MPPVs of $H_{n}, n=1,2, \ldots, 6$, the most probable depths $\left(D_{n}{ }^{*}, n=1\right.$, $2, \ldots, 5)$ of five internal boundaries for $N^{*}=6$ are determined as $1.28,5.16,6.77,8.43$, and $10.65 \mathrm{~m}$ (see Row 7 in Table 3), respectively. Similar procedures are also applied to other $N$ values besides $N=6$ to obtain their corresponding most probable depths $\left(D_{n}{ }^{*}, n=1,2, \ldots, N\right.$ 1) of internal boundaries, which are summarized in Table 3. For any $N$, the most probable depth of the lower boundary of the $N$-th soil layer is fixed at the testing depth, i.e., $D_{N}{ }^{*}=15 \mathrm{~m}$ in this example. Figure 9 shows an evolution of layer identification as $N$ increases from 1 to 10. As $N=1$, there is no internal boundary. The number of internal boundaries increases from one for $N=2$ to nine for $N=10$. For $N^{*}=6$, the five internal boundaries of the six soil layers are plotted by red solid lines in Figure 9. As a reference, Figure 9(a) shows the threshold values of $I_{c}$ between different SBTs by vertical red dashed lines in together with the 
$I_{c}$ profile (see open circles). Then, the respective soil types in the six soil layers in order from the ground surface to the depth of $15 \mathrm{~m}$ are determined, and they are classified as SBT 6 and 5, SBT 4, SBT 3, SBT 5, SBT 5, and SBT 5 (see Table 1 for detailed explanations of SBTs). It is found that although SBTs of soils below the depth of $7.0 \mathrm{~m}$ are similar, they are divided into three layers (i.e., the bottom three layers) because the $I_{c}$ in these three layers have different spatial variability along the depth. The spatial variability of $I_{c}$ is, explicitly and quantitatively, incorporated into soil stratification through random field modeling in the proposed approach.

More importantly, using the posterior samples of $H_{n}, n=1,2, \ldots, 6$, random samples of depths of the five internal layer boundaries are calculated, with which their standard deviations are obtained through conventional statistical analyses. The standard deviation of boundary depths quantitatively reflects the uncertainty in the location of soil layer boundaries. As shown in Figure 9(c), the fourth and fifth internal boundaries at the respective depths of 8.43 and $10.65 \mathrm{~m}$ (i.e., boundaries of the bottom three soil layers) have relatively large standard deviations (i.e., around $0.7 \mathrm{~m}$ ) in comparison with the top three boundaries. This might be attributed to the fact that the bottom three soil layers have similar SBTs, and the statistical differences in $I_{c}$ values within the bottom three soil layers are smaller than those in the top three layers. The proposed approach rationally quantifies the uncertainty in the location of soil layers boundaries identified based on the $I_{c}$ profile.

\section{Results comparison}

Figure 10 compares the soil stratification results from the proposed approach with those from other methods, such as FBSC approach (Zhang and Tumay, 1999), BSCS approach based on Robertson SBT chart (Wang et al. 2013), and WTMM method (Ching et al. 2015b). In general, the soil layers identified from the proposed approach are favorably comparable with 
those reported in these literatures. Note that the SBTs in Figure 10(a) and (b) are, respectively, determined by the $I_{c}$ value in each soil layer according to the threshold $I_{c}$ values summarized in Table 1 and by the location of data points $\left(F_{r}, Q_{t n}\right)$ in Robertson SBT chart without the need of calculating $I_{c}$. Hence, some differences in SBTs can be observed.

One difference in soil stratification results obtained from different approaches occurs at the second internal boundary identified from the proposed approach, which is located at the depth of $5.16 \mathrm{~m}$ (see Figure 10(a)). This boundary was not obtained from BSCS approach based on Robertson SBT chart (Wang et al. 2013) and FBSC approach (Zhang and Tumay 1999) (see the second and third columns of Figure 10(b), respectively), while a close boundary at the depth of around 5.0m was identified by WTMM method (Ching et al. 2015b), as shown by a dashed line in the first column of Figure 10(b). Based on the $I_{c}$ values, soils in the second and third layers identified from the proposed approach are classified as SBT 4 (silt mixtures: clayey silt to silty clay) and SBT 3 (clays: silty clay to clay), respectively, and the transformation between the two SBTs starts occurring at the depth of around $5.0 \mathrm{~m}$. Since both the proposed approach and WTMM method directly use $I_{c}$ as input, it is not surprising to see that one boundary at the depth of around $5.0 \mathrm{~m}$ is identified from the two approaches. In contrast, based on Robertson SBT chart and FBSC approach, soils in the two layers are classified as the same type of soil, i.e., SBT 3 (clays: silty clay to clay) and highly probable clayey soil (HPC), respectively. Hence, the layer boundary at the depth of around $5 \mathrm{~m}$ was not identified from BSCS approach based on Robertson SBT chart and FBSC approach.

Another difference in CPT-based soil stratification results is that one layer boundary was identified at the depth of around $13.0 \mathrm{~m}$ to divide the soils from the depth of $10.65 \mathrm{~m}$ to $15.0 \mathrm{~m}$ into two layers by WTMM method, as shown by the lower dashed line in the first column of Figure 10(b). The boundary does not appear in results from BSCS approach, FBSC approach, and the proposed approach. The above observations indicate that CPT-based soil 
stratification depends on the criterion adopted to discriminate soil layers. In comparison with the three approaches developed in the literature, the proposed approach provides not only the most probable soil stratigraphy but also quantifies the uncertainty in the identified soil stratigraphy (see Figures 8 and 9(c)), which allows incorporating uncertainty in soil stratigraphy into reliability-based analyses and designs of geotechnical structures (e.g., CPTbased deep foundation design). It shall, however, be pointed out that the proposed approach may take quite some computation time because an integral in Eq. (9) needs to be repetitively evaluated for calculating the likelihood function. Table 3 shows the computation time taken on a desktop computer with 8 GB RAM and one Intel Core i5 CPU clocked at $2.7 \mathrm{GHz}$ for each soil stratification model considered in the NGES example. For a given set of CPT data, the computation time of the proposed approach is affected by the number, $N$, of soil layers for a soil stratification model concerned and the number of samples generated in SuS.

Figure 10 also compares CPT-based soil stratification results (see Figures 10(a) and (b)) with that (see Figures 10(c)) based on boring $\log$ obtained at the clay site. It is obvious that soil stratification results from CPT-based approaches are inconsistent with those based on the boring log. Such inconsistence is attributed to different soil classification systems adopted in CPT-based approaches and boring log, which was also reported in the literature (Zhang and Tumay, 1999; Robertson, 2009; Wang et al. 2013). CPT-based soil classification is based on mechanical responses of soils during penetration and mainly reflects SBTs, but the soils in four layers according to the boring log are classified based USCS (Zhang and Tumay 1999; ASTM 2006) that classifies soils by the grain size distribution and the Atterberg limits and reflects soil compositions. This indicates that the soil stratigraphy inferred from site investigation data relies on soil classification systems (e.g., SBTs and USCS) adopted to determine the soil type profile. 


\section{Validation of the Proposed Approach Using a Simulated $I_{c}$ Profile}

The actual soil stratigraphy is unknown at a real site and can only be inferred from site investigation data and prior knowledge in practice. For further validation, the proposed approach is applied to identifying soil stratigraphy at a virtual site in this section, where the actual soil layer boundaries are known and can be used to simulate the $I_{c}$ profile for validating the proposed approach. As shown in Figure 11, the virtual site is comprised of five soil layers with respective thicknesses of 2, 3, 10, 20 and $15 \mathrm{~m}$ from the ground surface to the depth of $50 \mathrm{~m}$. Correspondingly, there are four internal boundaries located at depths of 2.0, $5.0,15.0$, and $35.0 \mathrm{~m}$, respectively. To model the spatial variability of $I_{c}$, the $I_{c}$ profile in the five soil layers is represented by five one-dimensional and mutually independent lognormal random fields, for which the correlation function is given by Eq. (2) and random field parameters are shown in Figure 11. Using the five lognormal random field models in the five soil layers, a profile of $I_{c}$ is simulated from the ground surface to the depth of $50 \mathrm{~m}$ at the interval of $0.05 \mathrm{~m}$, resulting in a total of $1000 I_{c}$ data points along the depth. Figure 12(a) shows one profile of $I_{c}$ simulated at the virtual site, and the figure also includes actual soil layer boundaries by horizontal red dashed lines as a reference. The $I_{c}$ profile shown in Figure 12(a) is used as input in the proposed approach to identify soil stratigraphy at the virtual site. Consider, for example, $N_{\max }=10$ again. Then, $N$ varies from 1 to 10 . In addition, $p_{0}$ and $N_{c s}$ are set as 0.1 and 10,000, respectively, to perform SuS for BUS.

Table 4 summarizes the soil stratification results (including $\ln p(\xi \mid N), \ln P(N \mid \xi)$, and $\left.D_{n}{ }^{*}, n=1,2, \ldots, N\right)$ obtained from the proposed approach for different $N$ values at the virtual site. The maximum value of $\ln p(\xi \mid N)$ (or, equivalently, $p(\xi \mid N)$ ) occurs at $N=5$, and its corresponding value of $\ln P(N \mid \xi)$ is around zero (i.e., $P(N \mid \xi) \approx 1)$. This is indicates that it is almost certain that the number of soil layers is equal to five based on the simulated $I_{c}$ profile at the virtual site, which is identical to the true number of soil layers at the site (see Figure 
11). Moreover, the four internal boundaries identified from the proposed approach are located at depths of 2.75, 5.02, 15.03, and $35.04 \mathrm{~m}$, as shown by horizontal red lines in Figure 12(b). These boundaries are close to their respective true boundaries at depths of 2.0, 5.0, 15.0, and $35.0 \mathrm{~m}$ (see horizontal red dashed lines in Figure 12(a)). Slight differences between the estimated depths and the true depths of internal boundaries might be attributed to the random fluctuation in the simulated $I_{c}$ profile. Moreover, Table 4 also provides the computation time for different $N$ values in this simulated example. In general, the computational time taken for a given $N$ value is less than that for the NGES example (see Table 3) because the number (i.e., $\left.N_{c s}=10,000\right)$ of samples generated in each level of $\mathrm{SuS}$ is much less in this simulated example.

Figure 12(c) shows the standard deviations of the depths of four internal boundaries obtained from the proposed approach. Results show that the standard deviations of depths (i.e., $D_{2}$ and $D_{3}$ ) of the second and third internal boundaries are less than those of the other two internal boundaries at depths of $D_{1}$ and $D_{4}$. It is not surprising to see this because the mean value (i.e., 1.8) of $I_{c}$ in the third layer are significantly different from the respective mean values (i.e., 2.7 and 3.0) of $I_{c}$ in the second and fourth layers (see Figure 11). In contrast, the differences (i.e., 0.5 and 0.4 ) in the mean values of $I_{c}$ in the first and second layers divided by $D_{1}$ and in the fourth and fifth layers divided by $D_{4}$ are relatively small, leading to relatively large identification uncertainty in $D_{1}$ and $D_{4}$. The identification uncertainty in soil layer boundaries provided by the proposed approach reflects statistical differences (e.g., such as the difference in the mean value) in $I_{c}$ values of adjacent soil layers. Nevertheless, further studies on factors affecting probabilistic soil stratification (including soil layer identification and uncertainty quantification) are warranted. 


\section{Summary and Conclusions}

This paper proposed a Bayesian framework for probabilistic soil stratification based on soil behaviour type index $I_{c}$ calculated from cone penetration test (CPT) measurements, in which the inherent spatial variability of $I_{c}$ along the depth is explicitly taken into account through random field modeling. The proposed Bayesian framework formulates the number $N$ and thicknesses $\underline{H}_{N}$ of soil layers that delineates soil stratigraphy as random variables to quantify their identification uncertainty based on the $I_{c}$ profile and prior knowledge. By this means, it not only provides the most probable soil stratigraphy identified from the $I_{c}$ profile and prior knowledge, but also quantifies the degrees-of-belief in the identified soil stratigraphy. Such information is summarized in the updated knowledge on $N$ and $\underline{H}_{N}$ obtained from the proposed Bayesian framework. To address the computational difficulties in Bayesian updating, a Subset Simulation (SuS)-based Bayesian updating technique, i.e., Bayesian Updating with Structural Reliability Method (BUS) using SuS, is used to obtain the updated knowledge on $N$ and $\underline{H}_{N}$ in this study. Using BUS with SuS, the model evidence $p(\xi \mid N)$ and posterior samples of $\underline{H}_{N}$ (equivalently, samples of boundary depths $\underline{D}_{N}$ ) for a given soil stratification model with $N$ layers are obtained by a single run of $\mathrm{SuS}$ for comparing different soil stratification models with different numbers of soil layers and for quantifying the uncertainty in soil layer boundaries, respectively.

Equations were derived for the proposed approach, and were illustrated and validated using real and simulated $I_{c}$ profiles. Results showed that the proposed approach properly identifies the most probable soil stratigraphy (including the most probable number and thicknesses of soil layers) and rationally quantifies the uncertainty in identified soil stratigraphy with the consideration of inherent spatial variability of $I_{c}$. This allows incorporating uncertainty in soil stratigraphy into subsequent reliability-based analyses and designs of geotechnical structures, such as CPT-based deep foundation designs. 
It was also found that the identification uncertainty in soil layer boundaries provided by the proposed approach reflects statistical differences (e.g., the difference in mean values) in $I_{c}$ values of adjacent soil layers. Comparing the results obtained from different soil stratification approaches indicated that soil stratigraphy inferred from site investigation data relies on both soil classification systems (e.g., SBTs and USCS) and soil stratification methods (e.g., Bayesian model identification, WTMM, and subjective engineering judgments). It is prudent to select a soil classification system deemed appropriate to geotechnical analyses and designs concerned and to perform soil stratification in a transparent and traceable manner.

\section{Acknowledgments}

This work was supported by the National Key R\&D Program of China (Project No. 2017YFC1501301), the National Natural Science Foundation of China (Project Nos. 51679174, 51579190, and 51779189), and Young Elite Scientists Sponsorship Program by CAST (Project No. 2017QNRC001). The financial support is gratefully acknowledged. 


\section{Reference:}

Ahmed, A., and Soubra, A. H. 2014. Probabilistic analysis at the serviceability limit state of two neighboring strip footings resting on a spatially random soil. Structural Safety, 49(8): 2-9.

Ang, A.H.-S., and Tang, W.H. 2007. Probability concepts in engineering: emphasis on applications to civil and environmental engineering. John Wiley and Sons, New York.

ASTM. 2006. Standard practice for classification of soils for engineering purposes (Unified Soil Classification System). ASTM standard D2487. ASTM International, West Conshohocken, $\mathrm{Pa}$.

ASTM. 2011. D2487-11. Standard practice for classification of soils for engineering purposes (Unified Soil Classification System). ASTM International, West Conshohocken, Pa.

Au, S. K., and Beck, J. L. 2003. Subset simulation and its application to seismic risk based on dynamic analysis. Journal of Engineering Mechanics, 129(8): 901-917.

Au, S. K., and Beck, J. L. 2001. Estimation of small failure probabilities in high dimensions by subset simulation. Probabilistic Engineering Mechanics, 16(4): 263-277.

Au, S. K., Cao, Z. J., and Wang, Y. 2010. Implementing advanced Monte Carlo simulation under spreadsheet environment. Structural Safety, 32 (5): pp. 281-292.

Au, S. K., and Wang, Y. 2014. Engineering Risk Assessment with Subset Simulation. John Wiley and Sons, Singapore.

Beck, J. L., and Au, S. K. 2002. Bayesian updating of structural models and reliability using Markov Chain Monte Carlo Simulation. Journal of Engineering Mechanics, 128(4): 380391.

Betz, W., Papaioannou, I., and Straub, D. 2014. Adaptive variant of the BUS approach to Bayesian updating. Proceedings of the 9th International Conference on Structural Dynamics, EURODYN, Porto, Portugal, MS18, pp. 3021-3028. 
Bishop, C. M. 2006. Pattern Recognition and Machine Learning, Springer Science \& Business Media, LLC, USA.

Briaud, J. L. 2000. "The National Geotechnical Experimentation Sites at Texas A\&M University: clay and sand. A Summary.” National Geotechnical Experimentation Sites, Geotechnical Special Publication No. 93, 26-51

Cao, Z.J., and Wang, Y. 2013. Bayesian approach for probabilistic site characterization using cone penetration tests. Journal of Geotechnical and Geoenvironmental Engineering, 139(2): 267-276.

Cao, Z.J., and Wang, Y. 2014. Bayesian model comparison and selection of spatial correlation functions for soil parameters. Structural Safety, 49: 10-17.

Cao, Z.J., Wang, Y., and Li, D.Q. 2016. Quantification of prior knowledge in geotechnical site characterization. Engineering Geology, 203:107-116.

Cetin, K.O. and Ozan, C. 2009. CPT-based probabilistic soil characterization and classification. Journal of Geotechnical and Geoenvironmental Engineering, 135(1): 84107.

Ching, J., and Chen, Y.C. 2007. Transitional Markov chain Monte Carlo Method for Bayesian model updating, model class selection, and model averaging. Journal of Engineering Mechanics, 133(7): 816-832.

Ching, J., Phoon, K.K., Chen, J.R., and Park, J.H. 2013. Robustness of constant LRFD factors for drilled shafts in multiple strata. Journal of Geotechnical and Geoenvironmental Engineering, 139(7): 1104-14.

Ching, J., Phoon, K.K., and Yang, J.J. 2015a. Role of redundancy in simplified geotechnical reliability-based design - a quantile value method perspective. Structural Safety, 55: $37-$ 48. 
Ching, J., and Wang, J.S. 2016. Application of the transitional Markov chain Monte Carlo algorithm to probabilistic site characterization. Engineering Geology, 203: 151-167.

Ching, J., Wang, J.S., Juang, C.H., and Ku, C.S. 2015b. Cone penetration test (CPT)-based stratigraphic profiling using the wavelet transform modulus maxima method. Canadian Geotechnical Journal, 52(12): 1993-2007.

Ching, J., Wu, S., and Phoon, K.K. 2015c. Statistical characterization of random field parameters using frequentist and Bayesian approaches. Canadian Geotechnical Journal, 53(2): 285-298.

Chiu, C.F., Yan, W.M. and Yuen, K. 2012. Estimation of water retention curve of granular soils from particle-size distribution-a Bayesian probabilistic approach. Canadian Geotechnical Journal, 49(9): 1024-1035.

Depina, I., Le, T.M.H., Eiksund, G., and Strøm, P. 2015. Cone penetration data classification with Bayesian mixture analysis. Georisk Assessment and Management of Risk for Engineered Systems and Geohazards, 10(1): 27-41.

DiazDelaO, F.A., Garbuno-Inigo, A., Au, S.K., and Yoshida, I. 2017. Bayesian updating and model class selection with subset simulation. Computer Methods in Applied Mechanics and Engineering, 317: 1102-1121.

Douglas, B.J., and Olsen, R.S. 1981. Soil classification using electric cone penetrometer. In Proceedings of the Symposium on Cone Penetration Testing and Experience, St. Louis, Mo., 26-30 October 1981. Edited by G.M. Norris and R.D. Holtz. Geotechnical Engineering Division, American Society of Civil Engineers, New York. pp. 209-227.

Eslami, A., and Fellenius, B. H. 1997. Pile capacity by direct CPT and CPTu methods applied to 102 case histories. Canadian Geotechnical Journal, 34(6): 886-904.

Fenton, G.A. 1999a. Estimation for stochastic soil models. Journal of Geotechnical and Geoenvironmental Engineering, 125(6): 470-485. 
Fenton, G.A. 1999b. Random field modeling of cpt data. Journal of Geotechnical and Geoenvironmental Engineering, 126(12): 486-498.

Ganju, E., Prezzi, M., and Salgado, R. 2017. Algorithm for generation of stratigraphic profiles using cone penetration test data. Computers and Geotechnics, 90: 73-84.

Hegazy, Y.A., and Mayne, P.W. 2002. Objective site characterization using clustering of piezocone data. Journal of Geotechnical and Geoenvironmental Engineering, 128(12): 986-996.

Houlsby, N.M.T., and Houlsby, G.T. 2013. Statistical fitting of undrained strength data. Géotechnique, 63(14): 1253-1263.

Huang, J., Fenton, G., Griffiths, D.V., Li, D., and Zhou, C. 2017a. On the efficient estimation of small failure probability in slopes. Landslides, 14(2): 491-498.

Huang, J., Zheng, D., Li, D., Kelly, R., and Sloan, S.W. 2017b. Probabilistic characterization of 2D soil profile by integrating CPT with MASW data. Canadian Geotechnical Journal, doi.org/10.1139/cgj-2017-0429.

Jefferies, M.G., and Davies, M.P. 1993. Use of CPTu to estimate equivalent SPT $\mathrm{N}_{60}$. Geotechnical Testing Journal, 16(4): 458-468.

Jung, B.-C., Gardoni, P., and Biscontin, G. 2008. Probabilistic soil identification based on cone penetration tests. Géotechnique, 58(7): 591-603.

Ku, C.S., Juang, C.H., and Ou, C. Y. 2010. Reliability of CPT $\mathrm{I}_{\mathrm{c}}$ as an index for mechanical behaviour classification of soils. Géotechnique, 60(11): 861-875.

Kurup, P.U., and Griffin, E.P. 2006. Prediction of soil composition from CPT data using general regression neural network. Journal of Computing in Civil Engineering, 20(4): 281-289. 
Li, D.Q., Qi, X.H., Phoon, K.K., Zhang, L.M., and Zhou, C.B. 2014. Effect of spatially variable shear strength parameters with linearly increasing mean trend on reliability of infinite slopes. Structural Safety, 49: 45-55.

Li, D.Q., Shao, K.B., Cao, Z.J., Tang, X.S., and Phoon, K.K. 2016a. A generalized surrogate response aided-subset simulation approach for efficient geotechnical reliability-based design. Computers and Geotechnics, 74: 88-101.

Li, D.Q., Xiao, T., Cao, Z.J., Zhou, C.B., and Zhang, L.M. 2016b. Enhancement of random finite element method in reliability analysis and risk assessment of soil slopes using subset simulation. Landslides, 13(2): 293-303.

Li, H.S. and $\mathrm{Au}$, S.K. 2010. Design optimization using subset simulation algorithm. Structural Safety, 32(6): 384-392.

Li, H.S. and Cao, Z.J. 2016. Matlab codes of Subset Simulation for reliability analysis and structural optimization. Structural and Multidisciplinary Optimization, 54(2): 391-410.

Li, J., Zhang, L., Cassidy, M.J., Huang, J., and Kelly, R. 2015. Probabilistic identification of soil stratification. Géotechnique, 66(1): 1-11.

Liao, T., and Mayne, P.W. 2007. Stratigraphic delineation by three-dimensional clustering of piezocone data. Georisk Assessment and Management of Risk for Engineered Systems and Geohazards, 1(1): 102-119.

Lunne, T., Robertson, P.K., and Powell, J.J.M. 1997. Cone penetration testing in geotechnical practice. Blackie Academic, EFSpon/Routledge, New York.

Mayne, P.W., Christopher, B.R., and DeJong, J. 2002. Subsurface investigationsGeotechnical site characterization. Rep. No. FHWA NHI-01-031, Federal Highway Administration, U.S. Dept. of Transportation, Washington, D.C.

Mathworks, Inc. 2017. MATLAB - the language of technical computing. Available from http://www.mathworks.com/products/matlab/. 
Olsen, R.S., and Mitchell, J.K. 1995. CPT stress normalization and prediction of soil classification. In Proceedings of International Symposium on Cone PenetrationTesting, CPT95, Linköping, Sweden. SGI Report 3:95. Vol. 2, pp.257-262.

Phoon, K.K., Ching, J., and Chen, J. R. 2013. Performance of reliability-based design code formats for foundations in layered soils. Computers and Structures, 126: 100-106.

Phoon, K.K., Quek, S.T., and An, P. 2003. Identification of statistically homogeneous soil layers using modified Bartlett statistics. Journal of Geotechnical and Geoenvironmental Engineering, 129(7): 649-659.

Phoon, K.K., Quek, S.T. and An, P. 2004. Geostatistical analysis of cone penetration test (CPT) sounding using. Canadian Geotechnical Journal, 41(41): 356-365.

Qi, X.H., and Li, D.Q. 2018. Effect of spatial variability of shear strength parameters on critical slip surfaces of slope. Engineering Geology, 239: 41-49

Robertson, P.K. 1990. Soil classification using the cone penetration test. Journal of Geotechnical and Geoenvironmental Engineering, 27(1): 984-986.

Robertson, P.K. 2009. Interpretation of cone penetration tests - a unified approach. Canadian Geotechnical Journal, 46(46): 1337-1355.

Robertson, P.K. 2016. CPT-based soil behaviour type (SBT) classification system - an update. Canadian Geotechnical Journal, 53(12): 1910-1927.

Robertson, P.K. and Wride, C.E. 1998. Evaluating cyclic liquefaction potential using the cone penetration test. Canadian Geotechnical Journal, 37(3): 270-271.

Robertson, P.K., and Campanella, R.G. 1983a. Interpretation of cone penetration tests. Part I: sand. Canadian Geotechnical Journal, 20(4): 718-733.

Robertson, P.K., and Campanella, R.G. 1983b. Interpretation of cone penetration tests. Part II: clay. Canadian Geotechnical Journal, 20(4): 734-745. 
Santoso, A.M., Phoon, K.K., and Quek, S.T. 2011. Modified metropolis-hastings algorithm with reduced chain correlation for efficient subset simulation. Probabilistic Engineering Mechanics, 26(2): 331-341.

Sivia, D.S., and Skilling, J. 2006. Data analysis: a Bayesian tutorial. Oxford University Press, New York.

Straub, D. 2011. Reliability updating with equality information. Probabilistic Engineering Mechanics, 26(2): 254-258.

Straub, D., and Papaioannou, I. 2015. Bayesian updating with structural reliability methods. Journal of Engineering Mechanics, 141(3): 04014134.

Straub, D., Papaioannou, I., and Betz, W. 2016. Bayesian analysis of rare events. Journal of Computational Physics, 314: 538-556.

Tumay, M.T., Abufarsakh, M.Y., and Zhang, Z. 2008. From Theory to Implementation of a CPT-Based Probabilistic and Fuzzy Soil Classification. From Research to Practice in Geotechnical Engineering Congress. ASCE.

Uzielli, M., Vannucchi, G., and Phoon, K.K. 2005. Investigation of correlation structures and weak stationarity using the CPT soil behaviour classification index. In Proceedings of the 9th International Conference on Structural Safety and Reliability, Rome, Italy.

Vanmarcke, E. 2010. Random fields : analysis and synthesis. Second (Revised and Expanded) Edition, World Scientific Publishing Company.

Wang, Y., Cao, Z.J., and Au, S.K. 2010a. Efficient Monte Carlo Simulation of parameter sensitivity in probabilistic slope stability analysis. Computers and Geotechnics, 37(7-8): $1015-1022$.

Wang, Y., Au, S.K., and Cao, Z.J. 2010b. Bayesian approach for probabilistic characterization of sand friction angles. Engineering Geology, 114 (3-4): 354-363. 
Wang, Y., Fu, C. and Huang, K. 2017. Probabilistic assessment of liquefiable soil thickness considering spatial variability and model and parameter uncertainties. Géotechnique, 67(3): $228-241$

Wang, Y., Huang, K., and Cao, Z. J. 2014. Bayesian identification of soil strata in london clay. Géotechnique, 64(3): 239-246.

Wang, Y., Huang, K. and Cao, Z. J. 2013. Probabilistic identification of underground soil stratification using cone penetration tests. Canadian Geotechnical Journal, 50(7): 766776.

Wickremesinghe, D.S., and Campanella, R.G. 1991. Statistical methods for soil layer boundary location using the cone penetration test. In Proceedings of the 6th ICASP, Mexico, Vol. 2, pp. 636-643.

Xiao, T., Li, D.Q., Cao, Z.J., and Zhang, L.M. 2018. CPT-Based Probabilistic Characterization of Three-Dimensional Spatial Variability Using MLE, ASCE Journal of Geotechnical and Geoenvironmental Engineering, 144(5), 04018023.

Xiao, T., Li, D.Q., Cao, Z.J., Au, S.K., and Phoon, K.K. 2016. Three-dimensional slope reliability and risk assessment using auxiliary random finite element method. Computers and Geotechnics, 79, 146-158.

Yan, W.M., Yuen, K.V., and Yoon, G.L. 2009. Bayesian probabilistic approach for the correlations of compression index for marine clays. Journal of Geotechnical and Geoenvironmental Engineering, 135(12): 1932-1940.

Yuen, K.V. 2010a. Recent developments of Bayesian model class selection and applications in civil engineering. Structural Safety, 32(5): 338-346.

Yuen, K.V. 2010b. Bayesian Methods for Structural Dynamics and Civil Engineering. John Wiley and Sons, Singapore. 
Zhang, Z., and Tumay, M.T. 1999. Statistical to fuzzy approach toward CPT soil classification. Journal of Geotechnical and Geoenvironmental Engineering, 126(6): 179186. 


\section{List of Figures}

Figure 1. Soil behaviour type chart and soil behaviour type index $I_{c}$ contours (Modified from Robertson 2009).

Figure 2. CPT data and soil behaviour type index $\left(I_{c}\right)$ values at the clay site of the NGES at Texas A\&M University (Modified from Zhang and Tumay (1999), C ASCE)

Figure 3. Random field modelling of the profile of soil behaviour type index

Figure 4. Illustration of the linear constraint of thicknesses of two layers within a depth of $15.0 \mathrm{~m}$

Figure 5. Flowchart for implementation of the proposed method

Figure 6. Boring log at the clay site of the NGES at Texas A\&M University (Modified from Zhang and Tumay (1999), (C) ASCE)

Figure 7. Logarithm of the evidence of soil stratification models with different numbers of soil layers

Figure 8. Posterior samples, marginal PDFs, and correlation coefficients of thicknesses of the most probable soil stratification model with $N^{*}=6$

Figure 9. Evolution of soil stratification results at the NGES clay site as $N$ varies from 1 to 10

Figure 10. Results comparison

Figure 11. A virtual site for simulating the $I_{c}$ profile

Figure 12. Comparison of soil stratification results with the true soil stratigraphy at the virtual site 


\section{List of Tables}

Table 1. Soil classification based on soil behaviour type index

Table 2. Typical ranges of random field parameters adopted in this study

Table 3. Soil stratification results from the proposed approach at the NGES clay site

Table 4. Soil stratification results from the proposed approach at the virtual site 
Table 1. Soil classification based on soil behaviour type index ${ }^{a}$

\begin{tabular}{ccc}
\hline Zone & Soil Behaviour Type & $I_{c}$ \\
\hline 2 & Organic soils: peats & $>3.6$ \\
3 & Clays: silty clay to clay & $2.95-3.6$ \\
4 & Silt mixtures: clayey silt to silty clay & $2.60-2.95$ \\
5 & Sand mixtures: silty sand to sandy silt & $2.05-2.6$ \\
6 & Sands: clean sand to silty sand & $1.31-2.05$ \\
7 & Gravelly sand to dense sand & $<1.31$ \\
\hline
\end{tabular}

Note $a$ : Modified from Robertson and Wride (1998)

Table 2. Typical ranges of random field parameters adopted in this study

\begin{tabular}{cccc}
\hline & $\mu_{n}$ & $\sigma_{n}$ & $\lambda_{n}$ \\
\hline Minimum & 0.52 & 0 & 0.1 \\
Maximum & 4.12 & 1.04 & 1.2 \\
Range & {$[0.52,4.12]$} & $(0,1.04]$ & {$[0.1,1.2]$} \\
\hline
\end{tabular}


Table 3. Soil stratification results from the proposed approach at the NGES clay site

\begin{tabular}{|c|c|c|c|c|c|c|c|c|c|c|c|c|c|}
\hline \multirow{2}{*}{$N$} & \multirow{2}{*}{$\begin{array}{l}\text { ln-evidence } \\
\ln p(\xi \mid N)\end{array}$} & \multirow{2}{*}{$\ln P(N \mid \xi)$} & \multicolumn{10}{|c|}{ Most probable depths of soil layer lower boundaries $D_{n}{ }^{*}, n=1,2, \ldots, N$} & \multirow{2}{*}{$\begin{array}{l}\text { Computation } \\
\text { time }^{a}\end{array}$} \\
\hline & & & $D_{1}{ }^{*}$ & $D_{2}{ }^{*}$ & $D_{3}^{*}$ & $D_{4}{ }^{*}$ & $D_{5}{ }^{*}$ & $D_{6}^{*}$ & $D_{7}^{*}$ & $D_{8}^{*}$ & $D_{9}^{*}$ & $D_{10}^{*}$ & \\
\hline 1 & 304.99 & -48.57 & 15.00 & & & & & & & & & & $0.04 \mathrm{~s}$ \\
\hline 2 & 317.91 & -35.64 & 8.15 & 15.00 & & & & & & & & & $2.7 \mathrm{~h}$ \\
\hline 3 & 329.43 & -24.13 & 1.26 & 6.82 & 15.00 & & & & & & & & $4.5 \mathrm{~h}$ \\
\hline 4 & 344.79 & -8.76 & 1.23 & 6.77 & 8.12 & 15.00 & & & & & & & $6.8 \mathrm{~h}$ \\
\hline 5 & 350.35 & -3.20 & 1.24 & 6.78 & 8.41 & 10.64 & 15.00 & & & & & & $8.0 \mathrm{~h}$ \\
\hline 6 & 352.93 & -0.63 & 1.28 & 5.16 & 6.77 & 8.43 & 10.65 & 15.00 & & & & & $10.6 \mathrm{~h}$ \\
\hline 7 & 352.70 & -0.86 & 1.20 & 5.18 & 6.78 & 8.48 & 10.68 & 13.31 & 15.00 & & & & $15.1 \mathrm{~h}$ \\
\hline 8 & 346.66 & -6.90 & 1.33 & 5.41 & 6.75 & 8.47 & 10.95 & 11.19 & 13.21 & 15.00 & & & $19.5 \mathrm{~h}$ \\
\hline 9 & 342.49 & -11.06 & 0.38 & 1.21 & 5.36 & 6.77 & 8.36 & 10.92 & 11.11 & 13.28 & 15.00 & & $17.9 \mathrm{~h}$ \\
\hline 10 & 337.11 & -16.44 & 0.42 & 1.29 & 5.56 & 6.73 & 6.83 & 8.89 & 10.92 & 11.11 & 13.27 & 15.00 & $18.9 \mathrm{~h}$ \\
\hline
\end{tabular}

Note $a$ : On a desktop computer with 8 GB RAM and one Intel Core i5 CPU clocked at $2.7 \mathrm{GHz}$, and $N_{c s}=100,000$

Table 4. Soil stratification results from the proposed approach at the virtual site

\begin{tabular}{|c|c|c|c|c|c|c|c|c|c|c|c|c|c|}
\hline \multirow{2}{*}{$N$} & \multirow{2}{*}{$\begin{array}{l}\text { ln-evidence } \\
\ln p(\xi \mid N)\end{array}$} & \multirow[b]{2}{*}{$\ln P(N \mid \xi)$} & \multicolumn{10}{|c|}{ Most probable depths of soil layer lower boundaries $D_{n}{ }^{*}, n=1,2, \ldots, N$} & \multirow{2}{*}{$\begin{array}{l}\text { Computation } \\
\text { time }^{a}\end{array}$} \\
\hline & & & $D_{1}{ }^{*}$ & $D_{2}{ }^{*}$ & $D_{3}{ }^{*}$ & $D_{4}{ }^{*}$ & $D_{5}{ }^{*}$ & $D_{6}{ }^{*}$ & $D_{7}{ }^{*}$ & $D_{8}{ }^{*}$ & $D_{9}{ }^{*}$ & $D_{10}{ }^{*}$ & \\
\hline 1 & 1179.30 & -128.12 & 50.00 & & & & & & & & & & $0.05 \mathrm{~s}$ \\
\hline 2 & 1264.48 & -42.95 & 35.01 & 50.00 & & & & & & & & & $2.6 \mathrm{~h}$ \\
\hline 3 & 1274.35 & -33.07 & 5.01 & 35.09 & 50.00 & & & & & & & & $2.5 \mathrm{~h}$ \\
\hline 4 & 1297.40 & -10.03 & 5.00 & 15.10 & 35.01 & 50.00 & & & & & & & $3.3 \mathrm{~h}$ \\
\hline 5 & 1307.43 & 0.00 & 2.75 & 5.02 & 15.03 & 35.04 & 50.00 & & & & & & $3.6 \mathrm{~h}$ \\
\hline 6 & 1301.09 & -6.34 & 2.07 & 5.00 & 15.04 & 17.28 & 35.01 & 50.0 & & & & & $2.6 \mathrm{~h}$ \\
\hline 7 & 1296.45 & -10.98 & 2.03 & 5.01 & 8.03 & 15.05 & 35.29 & 46.04 & 50.0 & & & & $2.1 \mathrm{~h}$ \\
\hline 8 & 1284.07 & -23.36 & 2.75 & 5.01 & 5.16 & 11.10 & 15.02 & 35.01 & 36.74 & 50.0 & & & $3.7 \mathrm{~h}$ \\
\hline 9 & 1284.07 & -23.36 & 2.02 & 4.60 & 5.01 & 15.01 & 16.41 & 23.20 & 28.58 & 35.02 & 50.0 & & $3.1 \mathrm{~h}$ \\
\hline 10 & 1279.12 & -28.31 & 2.03 & 5.01 & 5.14 & 8.37 & 15.03 & 18.91 & 20.50 & 22.37 & 35.24 & 50.0 & $3.7 \mathrm{~h}$ \\
\hline
\end{tabular}




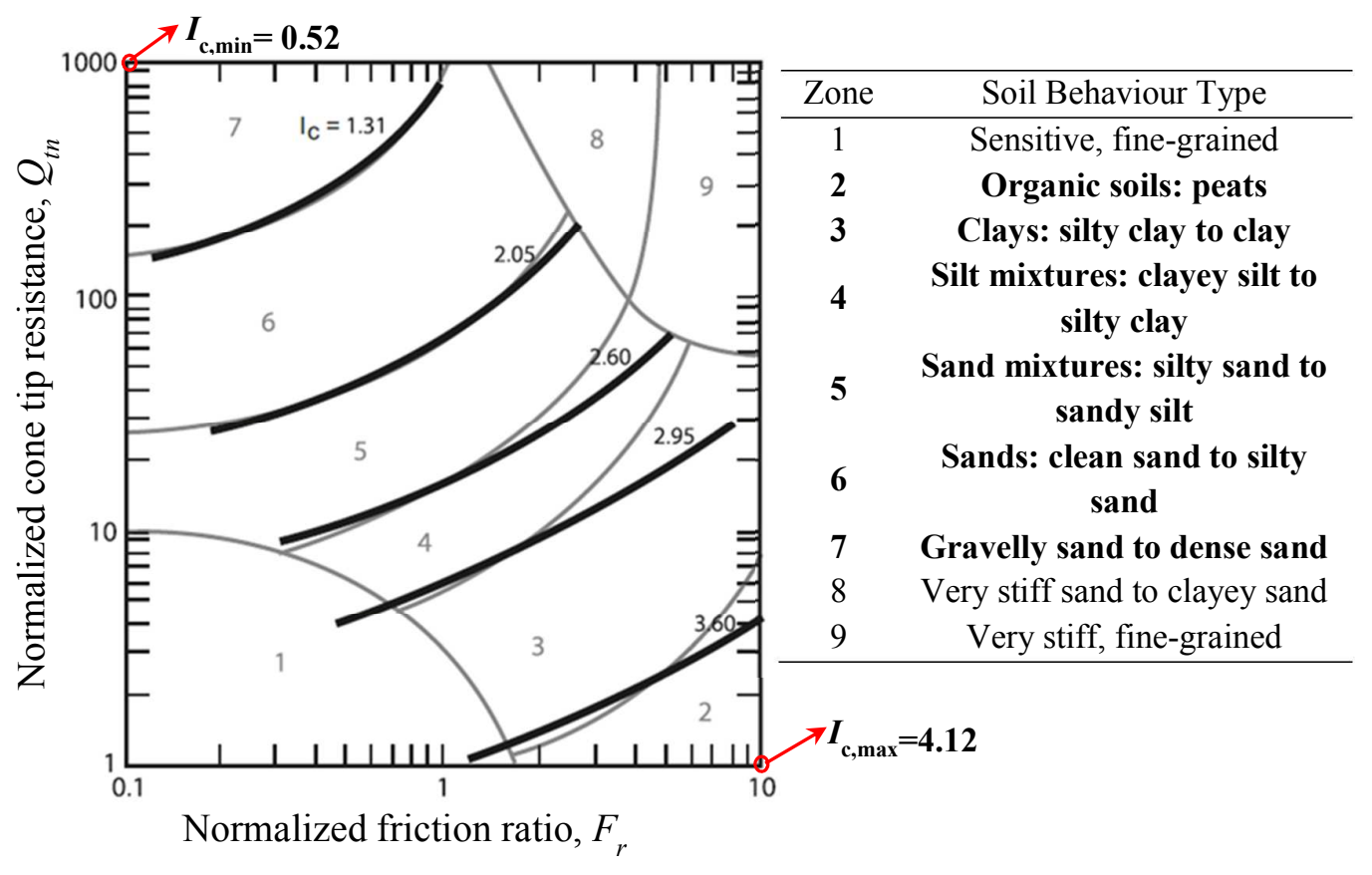

Figure 1. Soil behaviour type chart and soil behaviour type index $I_{c}$ contours (Modified from Robertson (2009)) 


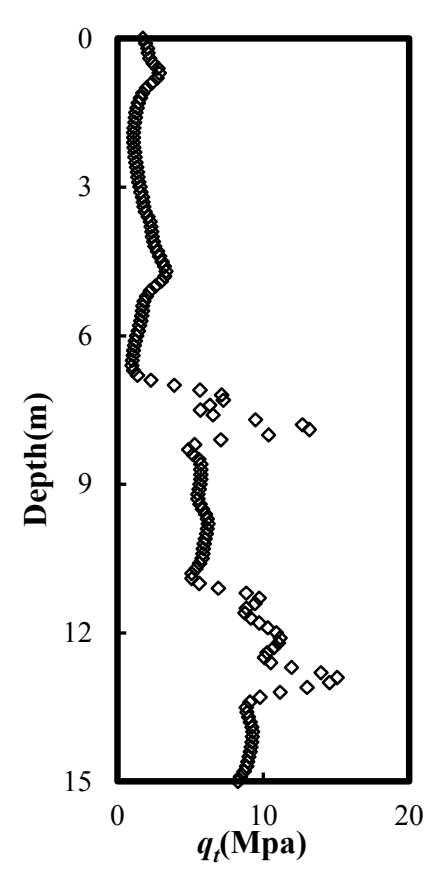

(a) $q_{t}$

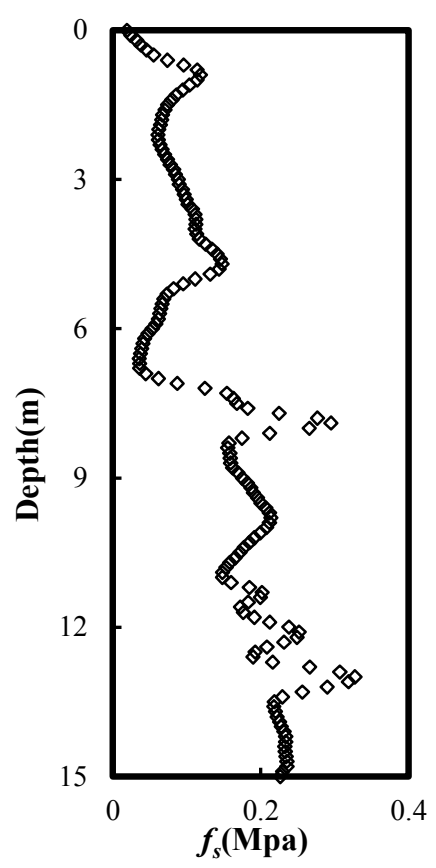

(b) $f_{s}$

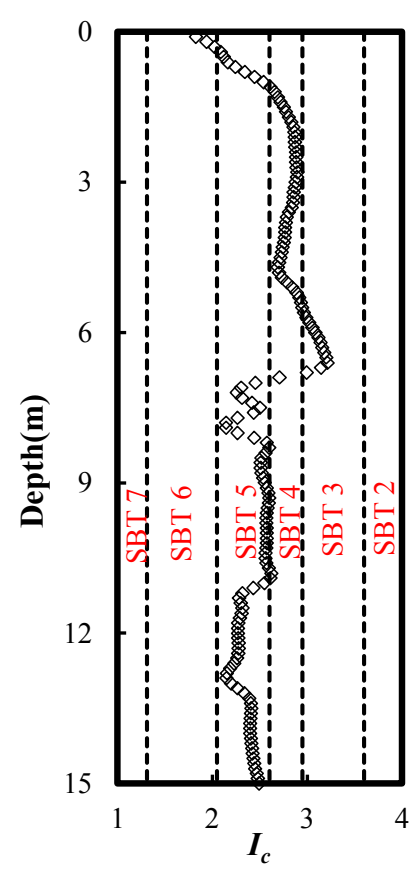

(c) $I_{c}$

Figure 2. CPT data and soil behaviour type index $\left(I_{c}\right)$ values at the clay site of the NGES at Texas A\&M University (Modified from Zhang and Tumay (1999), (C) ASCE) 


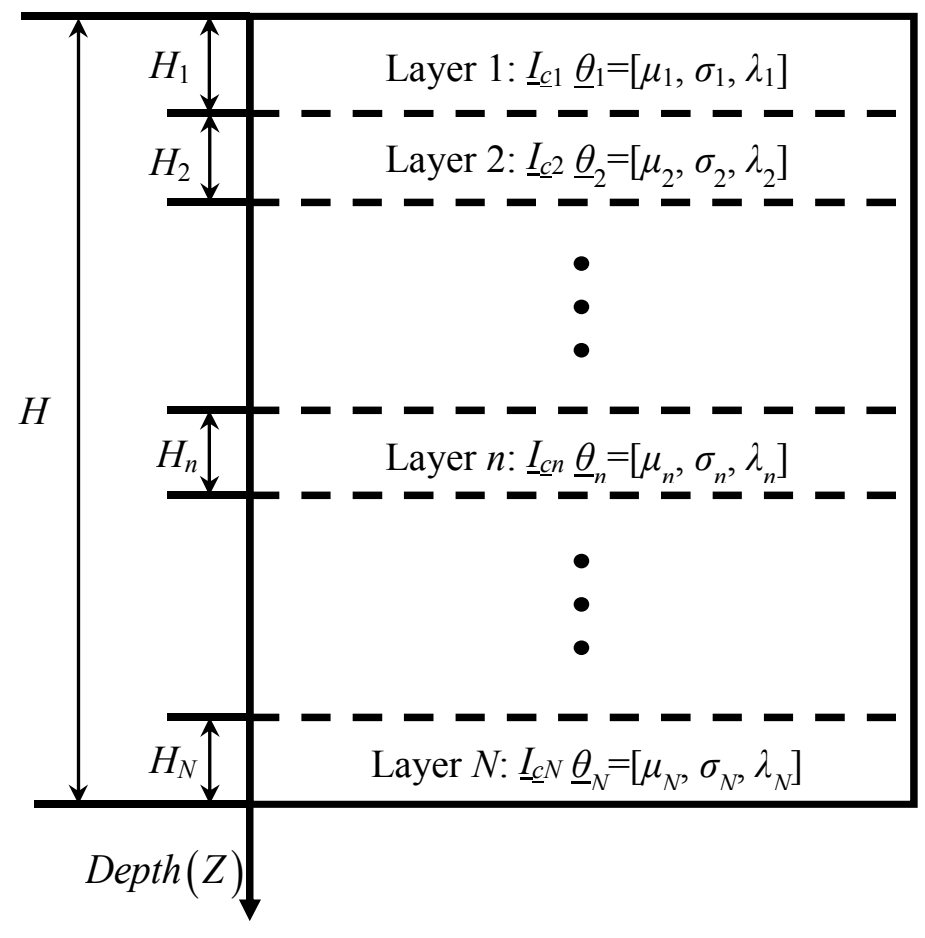

Figure 3. Random field modelling of the profile of soil behaviour type index 


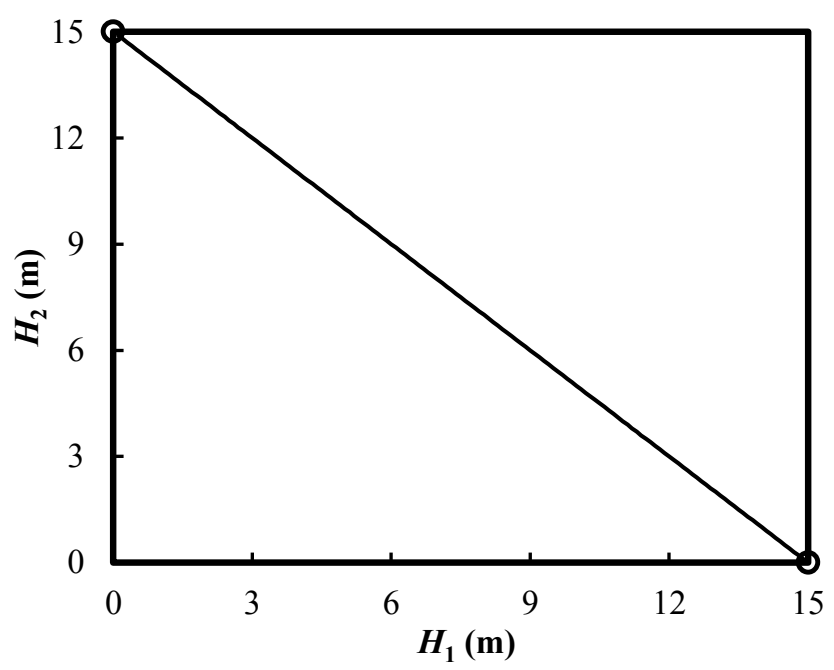

Figure 4. Illustration of the linear constraint of thicknesses of two layers within a depth of $15.0 \mathrm{~m}$ 
Obtain a set of CPT data and calculate the $I_{c}$ value at each testing depth using Eq.(1)

Choose a maximum number $N_{\max }$ of soil layers for the set of CPT data, leading to $N_{\max }$ possible values of $N$ varying from 1 to $N_{\max }$

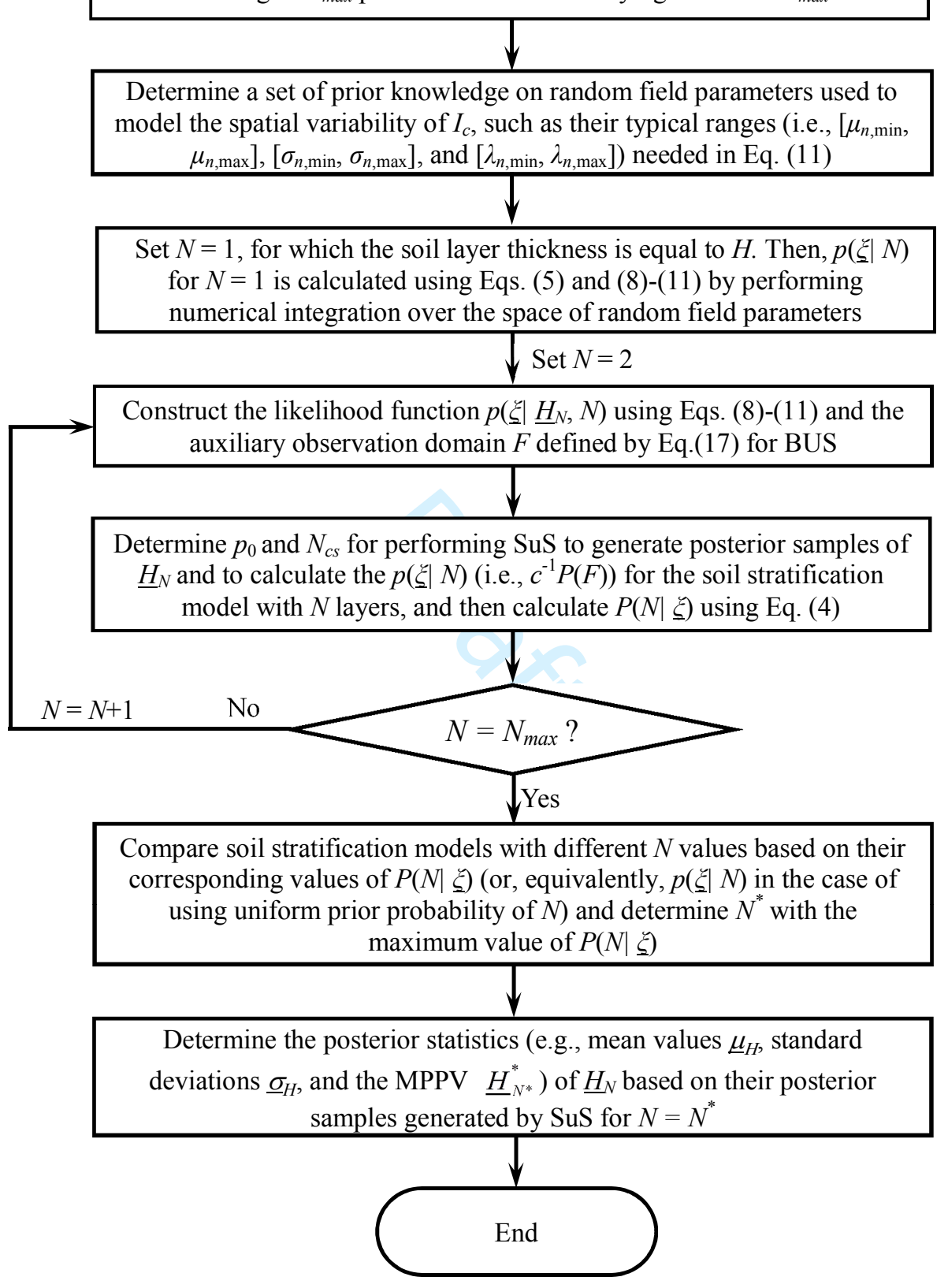

Figure 5. Flowchart for implementation of the proposed method 


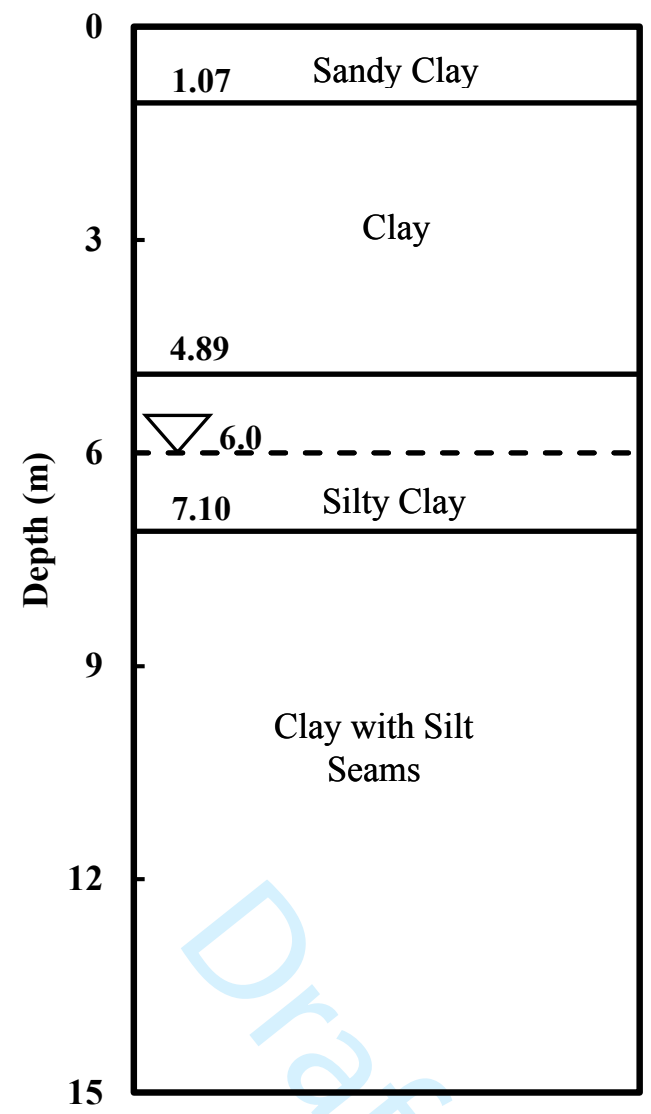

Figure 6. Boring $\log$ at the clay site of the NGES at Texas A\&M University (Modified from Zhang and Tumay (1999), (C) ASCE) 


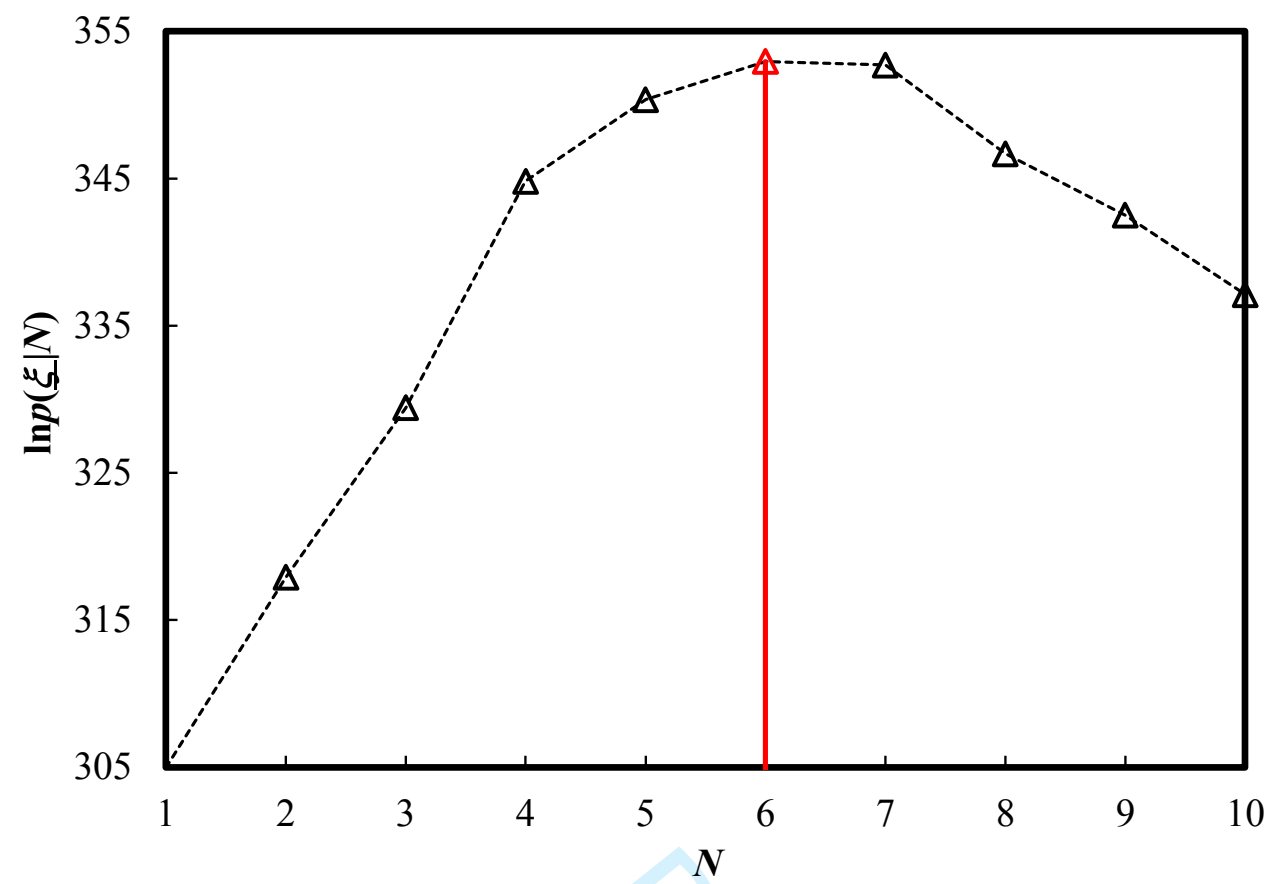

Figure 7. Logarithm of the evidence of soil stratification models with different numbers of soil layers 


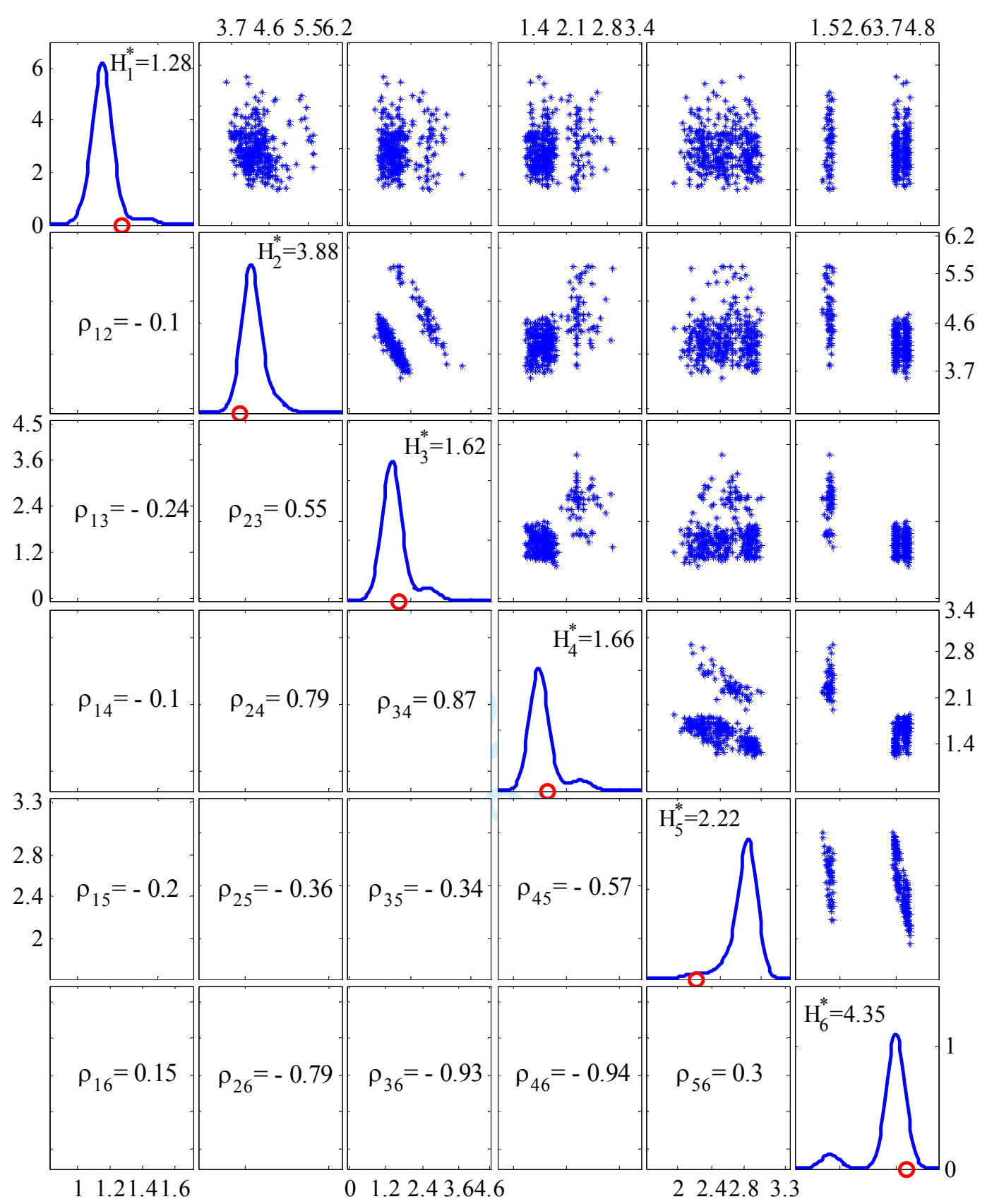

Figure 8. Posterior samples, marginal PDFs, and correlation coefficients of thicknesses of the most probable soil stratification model with $N^{*}=6$ 


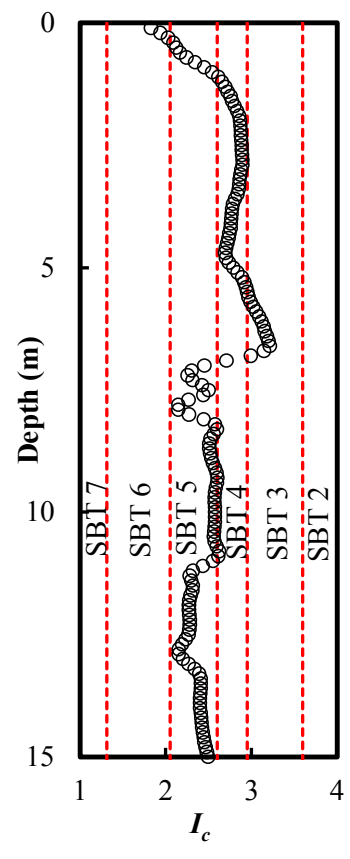

(a) $I_{c}$ profile at the NGES clay site

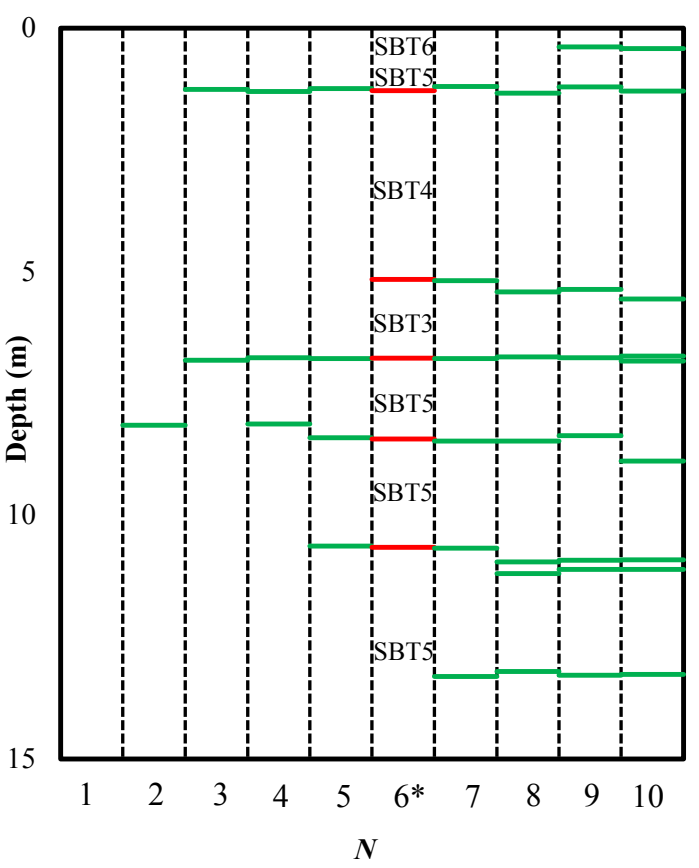

(b) Internal boundaries for different $N$ values

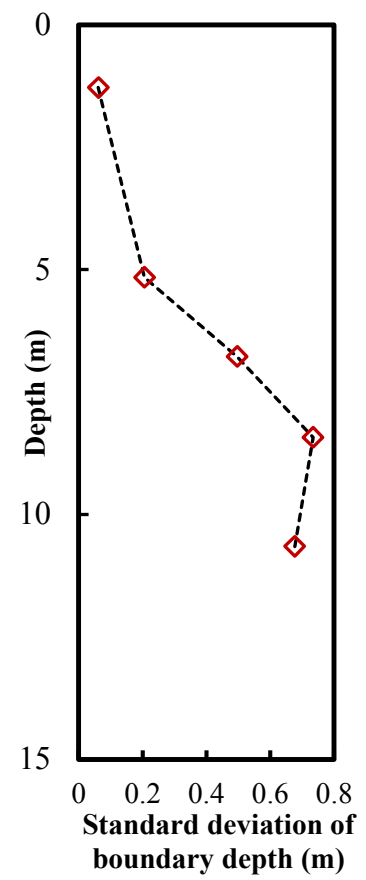

(c) Standard deviations of layer boundary depths

Figure 9. Evolution of soil stratification results at the NGES clay site as $N$ varies from 1 to 10 


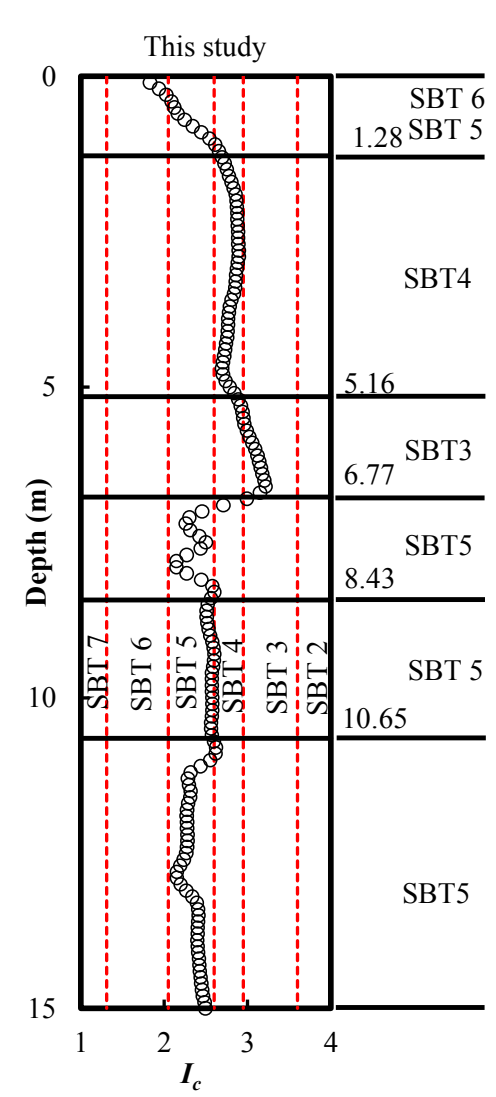

(a) This study

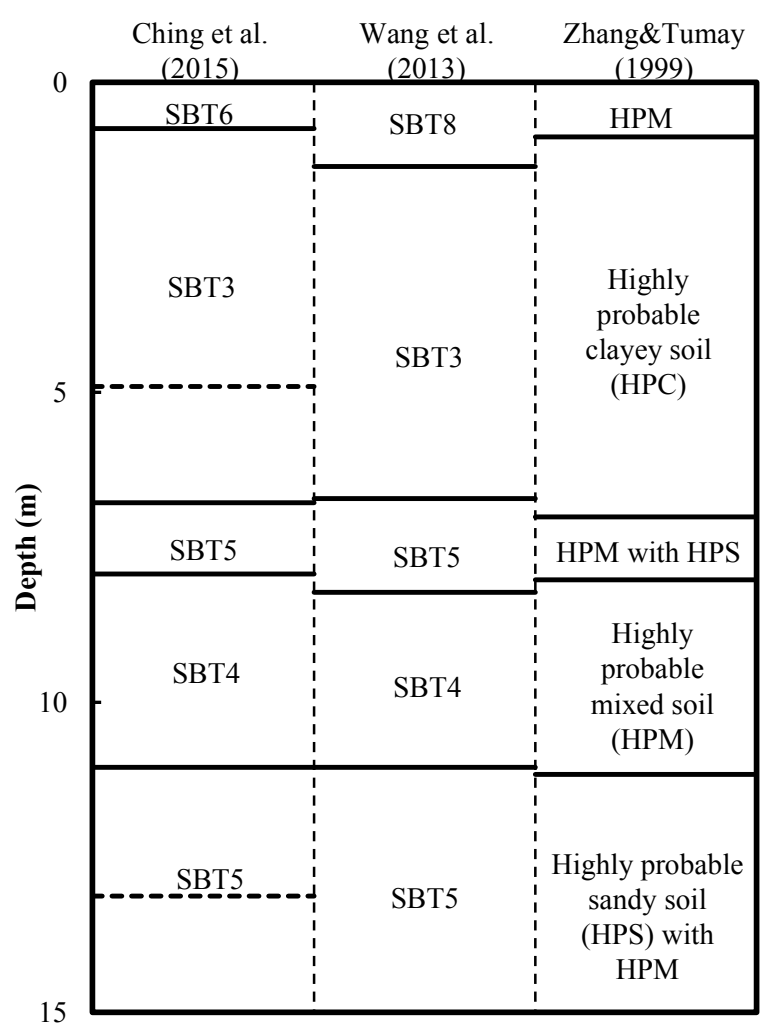

(b) Results reported in literature

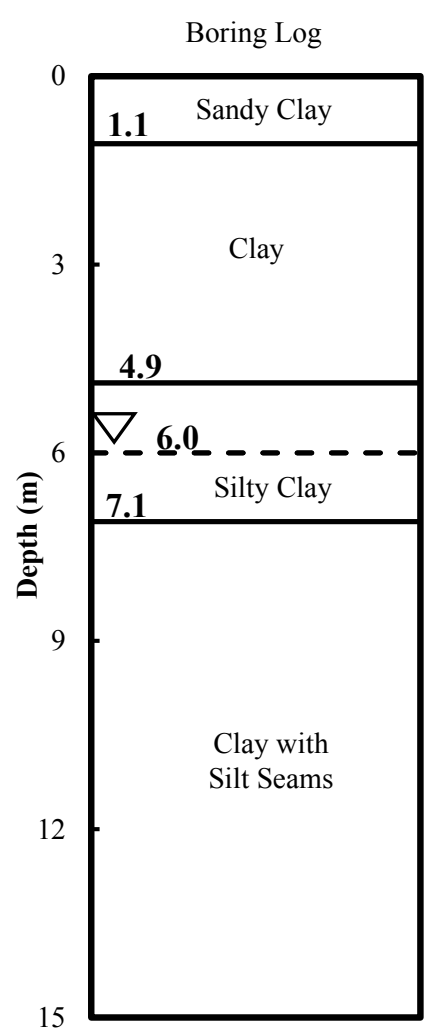

(c) Boring log

Figures 10. Results comparison 


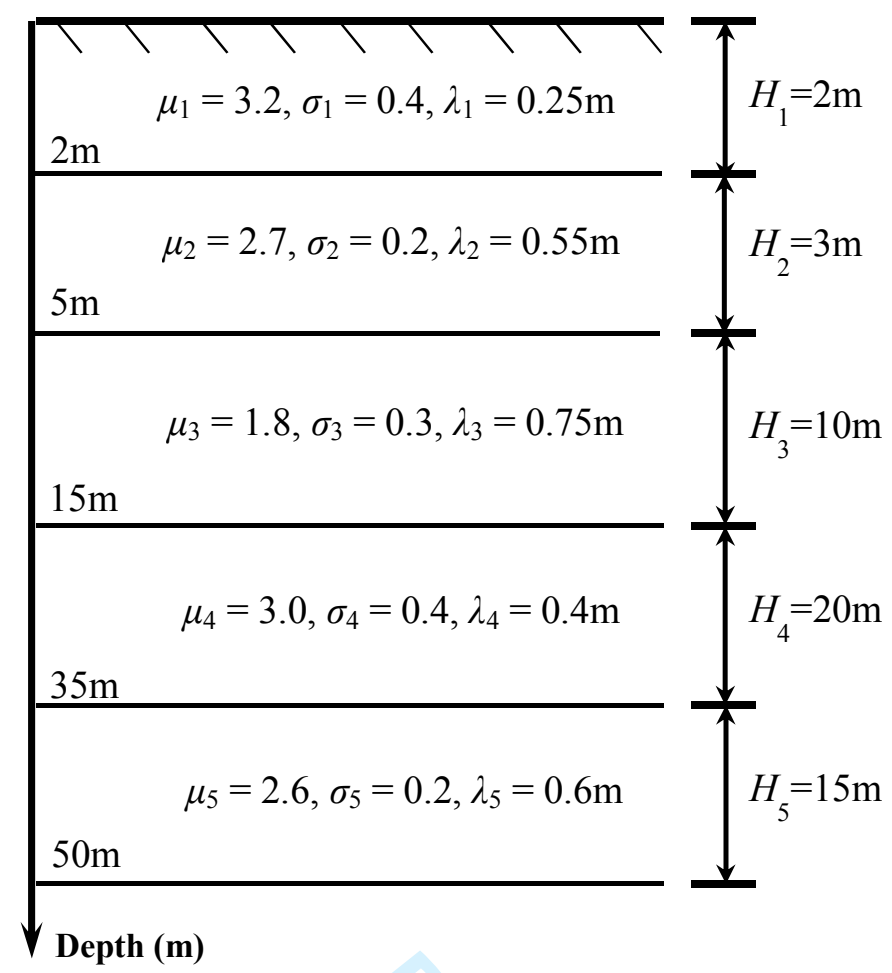

Figure 11. A virtual site for simulating the $I_{c}$ profile 


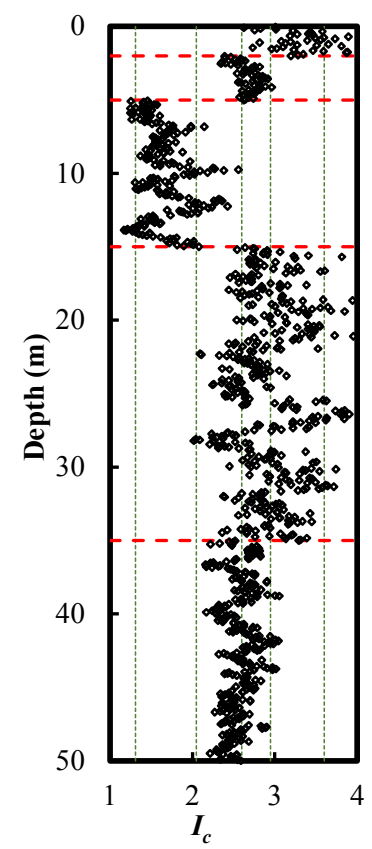

(a) Simulated $I_{c}$ profile

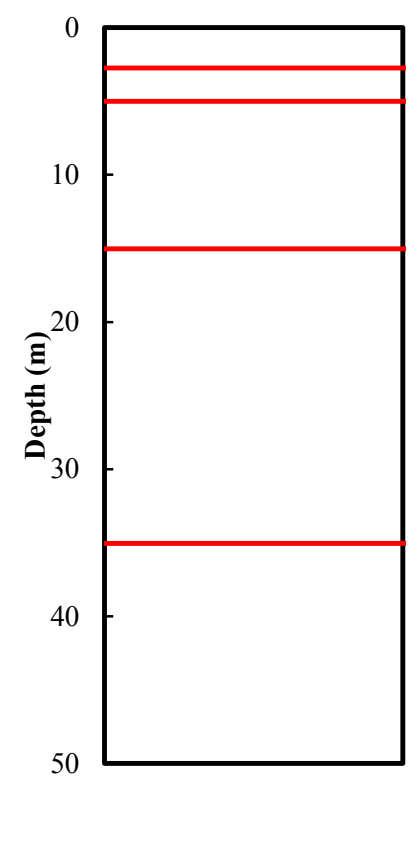

(b) Internal boundaries identified from the proposed approach

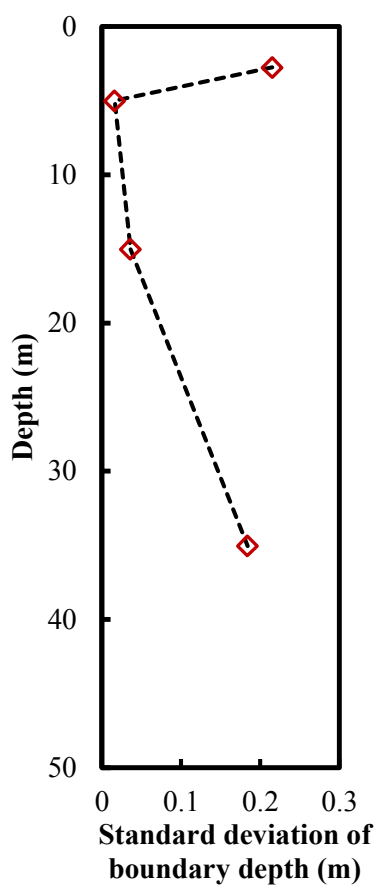

(c) Standard deviations of layer boundary depths

Figure 12. Comparison of soil stratification results with the true soil stratigraphy at the virtual site 